\title{
On the Effect of Outdated Channel Estimation in Variable Gain Relaying: Error Performance and PAPR
}

\author{
Zoran Hadzi-Velkov, Senior Member, IEEE, Diomidis S. Michalopoulos, Member, IEEE, \\ George K. Karagiannidis, Senior Member, IEEE, and Robert Schober, Fellow, IEEE
}

\begin{abstract}
For the conventional three-node amplify-andforward (AF) relaying setup, we investigate the effect of imperfect channel state information (CSI) at the relay on the overall performance. In particular, we consider variable gain (a.k.a, CSIassisted) AF relaying and derive expressions for the outage and the error probability for the case where the relay gain is adjusted based on outdated estimates of the source-relay channel, when operating over Nakagami- $m$ fading. For the case of Rayleigh fading in the source-relay link, we show that the results can be extended to the versatile case of imperfect CSI, where the estimation error is caused either by additive white Gaussian noise or by quantization noise. The obtained expressions are functions of the correlation coefficient between the actual sourcerelay channel and its corresponding estimate. We also optimize the power allocation for minimization of the outage probability under a total transmit power constraint. Numerical results reveal a considerable degradation of the overall performance, when CSI acquisition is not perfect. Moreover, it is shown that the average relay transmit power is affected when the CSI is outdated, a fact which impacts the design of variable gain relaying in practice. Since outdated CSI leads to fluctuations of the relay transmit power, we derive expressions for the complementary cumulative distribution function (CCDF) of the peak-to-average-power ratio (PAPR) at the relay. By comparing the error probability and the CCDF of the PAPR of variable gain relaying with those of fixed gain relaying, we shed some light onto the following question: How reliable has the instantaneous CSI at the relay to be for variable gain relaying to be preferable over fixed gain relaying, which requires only statistical CSI?
\end{abstract}

Index Terms-Wireless relaying, imperfect channel estimation, Nakagami-m fading, outage and error probability, peak-toaverage power ratio (PAPR).

Manuscript received January 15, 2012; revised July 22, 2012; accepted December 13, 2012. The associate editor coordinating the review of this paper and approving it for publication was Y. Mao.

This paper has been presented in part at the IEEE International Conference on Communications 2012, and the International ITG Conference on Systems, Communications and Coding 2013.

Z. Hadzi-Velkov is with the Faculty of Electrical Engineering and Information Technologies, Ss. Cyril and Methodius University, 1000 Skopje. $\mathrm{He}$ is currently visiting the Institute for Digital Communication at the Friedrich-Alexander University of Erlangen-Nuremberg, Germany, under the Humboldt Fellowship Program for Experienced Researchers (e-mail: zoranhv@ feit.ukim.edu.mk).

D. S. Michalopoulos and R. Schober are with the Department of Electrical and Computer Engineering, University of British Columbia, Vancouver, BC V6T1Z4, Canada, (e-mail: \{dio, rschober\}@ece.ubc.ca).

G. K. Karagiannidis is with the Wireless Communications Systems Group (WCSG), Department of Electrical and Computer Engineering, Aristotle University of Thessaloniki, GR-54124 Thessaloniki, Greece (e-mail: geokarag@auth.gr).

Digital Object Identifier 10.1109/TWC.2013.011513.120088

\section{INTRODUCTION}

W IRELESS relaying technology is included in the standards of future wireless networks, such as the Long Term Evolution-Advanced (LTE-A) system, due to a number of advantages that it promises in terms of system coverage and performance [1]. By using relays, terrestrial networks are able to extend their coverage to distant areas. The distributed nature of wireless relaying allows to combat not only smallscale fading, as conventional diversity techniques do, but also large-scale fading.

Amplify and forward (AF) relaying, which refers to simple amplification and forwarding of the information sent from a source terminal to a destination terminal, is considered to be the simplest implementation of wireless relaying in practice, since no other form of processing at the relay is required. Among the several variations of $\mathrm{AF}$ relaying, the most common one is so-called variable-gain relaying (VGR) ${ }^{1}$, where the gain employed at the relay compensates the fading of the source-relay link, aiming at maintaining a constant relay transmit power. In order to do so, the relay has to monitor the source-relay channel, such that the amplification gain can be adjusted according to the obtained channel state information (CSI). In this respect, the majority of the works in the literature assume perfect CSI acquisition (see, e.g., [2]-[5] and the references therein). Of particular interest is the work in [5], where it was shown that the gain policy that compensates for the source-relay channel leads to optimal performance of AF VGR, provided, however, that the CSI available at the relay is perfect.

In this paper, we study the effect of outdated channel estimation at the relay in a typical dual-hop (three-node) AF VGR system, operating in Nakagami- $m$ fading channels. Here, the term "outdated" refers to the case where the CSI is correct at the time of estimation, but corresponds to a different channel realization than the channel realization at the time it is used (see, e.g., [7]). As a result, outdated CSI pertains to a subset of the practical scenarios that are associated with the versatile term "imperfect" CSI. In this regard, we show that, for Rayleigh fading in the source-relay link, our analysis can be extended to the cases where the estimation error is caused by additive white Gaussian noise (AWGN) or by quantization

\footnotetext{
${ }^{1}$ VGR is also known in the literature as "channel state information (CSI)assisted" gain relaying.
} 
noise, covering thus a wide range of practical scenarios of imperfect CSI at the relay.

It is noted that this is not the first time that outdated CSI is considered in the context of wireless relaying [7]-[15]. However, the works [7]-[12] examine the effect of outdated CSI on various relay selection schemes (e.g., best, opportunistic or partial) in multiple-relay systems, where the outdated CSI causes potentially suboptimal relay selection. The effects of outdated CSI on two-way relaying and beamforming are studied in [13] and [15], respectively. Nevertheless, although the CSI used for relay selection is outdated, these works inherently assume both the selected relay and the destination have perfect CSI.

Here, we relax the above assumption of perfect CSI at both the relay and destination. We ground our argument on the fact that the channel estimation is typically realized by using pilot symbols transmitted periodically by the source, where the pilot insertion period depends on the channel coherence time of the end-to-end channel. The source-relay channel estimate at the relay is needed only to set its gain $G$ [2]. In order to conserve energy and to reduce complexity, the relay may estimate the source-relay channel infrequently, i.e., the channel estimation rate at the relay may be insufficient to capture some rapid source-relay channel fluctuations. Thus, although the relay may actually estimate the channel perfectly, these estimates may be outdated by the time they are used to amplify the incoming signal samples, because the actual source-relay channel may change rapidly between two successive channel estimates. In addition, it is also possible that the relay acquires imperfect CSI in cases where the CSI of the source-relay link is fed back to the relay from another terminal which could be, for instance, a central unit with global CSI knowledge. In such case, the relay may obtain limited feedback CSI, which suffers from quantization noise ${ }^{2}$. Consequently, in the above scenarios the relay's gain $G$ is set to a value that does not perfectly compensate the source-relay channel. This imperfect CSI at the relay does not necessarily affect the quality of channel estimation at the destination, as highly accurate (perfect) channel estimates are necessary for ideal coherent demodulation, as well as some higher layer functions, such as scheduling. Thus, the destination may update its CSI estimation more frequently than the relay.

The level of CSI imperfection at the relay is quantified through the power correlation coefficient between the actual and the estimated channel values, $\rho$. The performance metrics considered in this work are the end-to-end outage probability $(\mathrm{OP})$, the average bit error probability (ABEP), and the complimentary cumulative distribution function (CCDF) of the peak-to-average-power ratio (PAPR) at the output of the relay. It is noted that, as far as we are aware, this is the first time that the latter metric is used for the analysis of AF relaying schemes with imperfect CSI at the relay ${ }^{3}$. Our motivation behind using this metric is that, contrary to VGR schemes

\footnotetext{
${ }^{2}$ We thank one of the anonymous reviewers for bringing this scenario to our attention.

${ }^{3}$ We note that the CCDF of the relay transmit power is also studied in [16]. However, in [16] the fluctuations of the relay transmit power were the result of the pulse shaping process along with noise, and the case of imperfect CSI acquistion at the relay was not considered.
}

with perfect CSI, the relay transmit power in VGR experiences fluctuations when the CSI used for calculation of the relay gain is imperfect. The CCDF of the PAPR can be thus used to obtain the probability with which the power amplifier at the relay operates in its non-linear region, therefore distorting the output signal (see, e.g., [6]).

Apart from VGR, a common technique adopted in the literature is so-called fixed-gain relaying (FGR). In FGR, the relay gain is based only on the statistical (i.e., long-term) CSI of the source-relay channel, instead of the instantaneous CSI, rendering FGR a simpler alternative to VGR. By its nature, VGR has an advantage over FGR if the CSI is perfect, since on the one hand, it has a unit PAPR, and on the other hand, it results in lower outage and error probabilities [19]. What happens, however, when the CSI at the relay is outdated? One would expect that the advantage of VGR over FGR is lost when the CSI is not reliable. Indeed, our results show that there exists a correlation coefficient threshold, below which FGR outperforms VGR. This threshold exists not only for the outage and error probabilities, but also for the CCDFs of the PAPR. Hence, considering the relative simplicity of FGR, the above results shed some light onto which relaying scheme is preferable in scenarios where the CSI at the relay is outdated, yet its reliability is known.

\section{System AND Channel Model}

Let us consider a dual-hop wireless communication system, consisting of a source $S$, a VGR AF relay $R$, and a destination $D$. Each of the three nodes is equipped with a single antenna. The actual (complex-valued) coefficients of the $S-R$ and $R-D$ channels are denoted by $c_{X}$ and $c_{Y}$, respectively. The fading amplitudes, $\alpha=\left|c_{X}\right|$ and $\beta=\left|c_{Y}\right|$, are assumed to follow the Nakagami- $m$ distribution with fading parameters $m_{X}$ and $m_{Y}$, and mean squared values $E\left\langle\left|c_{X}\right|^{2}\right\rangle=E\left\langle\alpha^{2}\right\rangle=\Omega_{X}$ and $E\left\langle\left|c_{Y}\right|^{2}\right\rangle=E\left\langle\beta^{2}\right\rangle=\Omega_{Y}$, respectively. Here, $E\langle\cdot\rangle$ denotes expectation.

\section{A. Case of Outdated CSI}

Let us assume that the relay uses pilot symbols to estimate the time-varying $S$ - $R$ channel, but it repeats the estimation process not frequently enough to prevent channel samples from being outdated. Then, the relay's amplification factor $G$ is set based on an outdated estimate of $\alpha$, denoted by $\hat{\alpha}$. That is,

$$
G^{2}=\frac{E_{R}}{E_{S} \hat{\alpha}^{2}+N_{0}}
$$

where $N_{0}$ is the AWGN power, which is assumed identical for all links, and $E_{S}$ is the source's transmit power.

In (1), $E_{R}$ is a constant that equals the relay's transmit power when the CSI at the relay is perfect. As shown later in Section IV, the relay's transmit power under outdated CSI does not necessarily equal $E_{R}$. Furthermore, $\hat{\alpha}$ is an accurate channel estimate at the sampling instant, but it is already outdated when applied for setting $G$, due to the timevarying nature of the $S-R$ channel [7], [8], [14]. Thus, $\alpha$ and $\hat{\alpha}$ have identical statistical parameters; their correlation is a function of the estimation repetition rate and the maximum Doppler frequency of the $S$-R channel. Consequently, $\alpha$ and 
$\hat{\alpha}$ follow the Nakagami- $m$ distribution with identical shape parameter $m_{\hat{X}}=m_{X}$ and identical mean squared value $E\left\langle\alpha^{2}\right\rangle=E\left\langle\hat{\alpha}^{2}\right\rangle=\Omega_{X}$. The power correlation coefficient between $\alpha$ and $\hat{\alpha}$ is denoted by $\rho$.

Using pilot symbols sent by the source and forwarded by the relay, the destination is assumed to perfectly estimate the endto-end channel, performing thus ideal coherent demodulation. The instantaneous SNR at $D$ is given by

$$
\gamma=\frac{E_{S} \alpha^{2} \beta^{2} G^{2}}{\beta^{2} G^{2} N_{0}+N_{0}} .
$$

Combining (1) and (2) and neglecting the $N_{0}$ term, we obtain

$$
Z=\frac{X Y}{\hat{X}+Y},
$$

where $Z=\gamma / \Gamma_{0}, \Gamma_{0}=E_{S} / N_{0}, X=\alpha^{2}, \hat{X}=\hat{\alpha}^{2}$, and $Y=$ $\beta^{2}{ }^{4}$ Parameter $\Gamma_{0}$ is referred to as the transmit SNR. We note that Eq. (3) is actually an upper bound on the instantaneous SNR at $D$, yielding performance bounds on the ABEP and OP derived in Section III. This approximation is commonly used in the literature (see e.g. [3], [9]), since, as shown in Section VII, it yields highly accurate results, especially in the medium and high SNR region.

Random variables (RVs) $X, \hat{X}$, and $Y$ follow Gamma distributions with shape parameters $m_{X}, m_{\hat{X}}$, and $m_{Y}$, respectively, and scale parameters $E\langle X\rangle=\Omega_{X} / m_{X}, E\langle\hat{X}\rangle=$ $\Omega_{\hat{X}} / m_{\hat{X}}$, and $E\langle Y\rangle=\Omega_{Y} / m_{Y}$, respectively. Their probability density functions (PDFs) and cumulative density functions (CDFs) are given by

$$
\begin{gathered}
f_{U}(x)=\left(\frac{m_{U}}{\Omega_{U}}\right)^{m_{U}} \frac{x^{m_{U}-1}}{\Gamma\left(m_{U}\right)} \exp \left(-\frac{m_{U} x}{\Omega_{U}}\right), \quad x \geq 0, \\
F_{U}(x)=1-\frac{1}{\Gamma\left(m_{U}\right)} \Gamma\left(m_{U}, \frac{m_{U} x}{\Omega_{U}}\right), \quad x \geq 0,
\end{gathered}
$$

respectively, where $U \in\{X, \hat{X}, Y\}, \Gamma(\cdot)$ denotes the Gamma function defined in [22, Eq. (8.310.1)], and $\Gamma(\cdot, \cdot)$ is the incomplete Gamma function, defined in [22, Eq. (8.350.2)].

Since $m_{\hat{X}}=m_{X}$ and $\Omega_{X}=\Omega_{\hat{X}}$, owing to the outdated CSI assumption, the joint PDF of $X$ and $\hat{X}$ is given by [20, Eq. (126)]

$$
\begin{aligned}
f_{X \hat{X}}(x, \hat{x}) & =\left(\frac{m_{X}}{\Omega_{X}}\right)^{m_{X}+1} \frac{1}{(1-\rho) \Gamma\left(m_{X}\right)}\left(\frac{x \hat{x}}{\rho}\right)^{\frac{m_{X}-1}{2}} \\
& \times e^{-\frac{m_{X}(x+\hat{x})}{(1-\rho) \Omega_{X}}} I_{m_{X}-1}\left(\frac{2 m_{X} \sqrt{\rho x \hat{x}}}{(1-\rho) \Omega_{X}}\right)
\end{aligned}
$$

where $f_{A B}(\cdot, \cdot)$ denotes the joint PDF of RVs $A$ and $B$, $I_{m_{X}-1}(\cdot)$ is the $\left(m_{X}-1\right)$ th order modified Bessel function of the first kind defined in [22, Eq. (8.406.1)], and $\rho \in[0,1)$.

\section{B. Case of Gaussian Estimation Error}

Let us now consider the case of Rayleigh fading in the $S$ - $R$ link. Here, we show that the analysis based on the outdated CSI model presented above can also accommodate scenarios where the channel estimation error at the relay is caused by AWGN or by quantization noise.

\footnotetext{
${ }^{4}$ Strictly speaking, $Y$ is defined as $Y=\beta^{2} E_{R} / E_{S}$. However, since $\Omega_{Y}$ is arbitrarily chosen, the coefficient $E_{R} / E_{S}$ can be absorbed into $\Omega_{Y}$.
}

Let us express the estimate of channel $c_{X}, \hat{c}_{X}$, as $\hat{c}_{X}=$ $c_{X}+e$, where $c_{X}$ is a complex Gaussian $\mathrm{RV}$ and $e$ denotes the estimation error. For scenarios where $e$ represent AWGN or quantization noise, $e$ is also a complex Gaussian RV with zero mean and variance $\sigma_{e}^{2}$ [26]. In case of noisy estimation, $\sigma_{e}^{2}$ equals the AWGN power; in case of quantized CSI, $\sigma_{e}^{2}$ is determined by the distortion-rate function for a given rate [26].

The power correlation coefficient between $\hat{c}_{X}$ and $c_{X}$ is derived as

$$
\rho=\frac{E\left\langle\left|c_{X}\right|^{2}\right\rangle}{E\left\langle\left|\hat{c}_{X}\right|^{2}\right\rangle}=\frac{\Omega_{X}}{\Omega_{X}+\sigma_{e}^{2}} .
$$

Contrary to the outdated CSI model considered in Section II-A, $E\left\langle\left|\hat{c}_{X}\right|^{2}\right\rangle$ for the case of Gaussian estimation error does not equal $E\left\langle\left|c_{X}\right|^{2}\right\rangle$. However, it is emphasized that the analysis assuming the outdated CSI model can still be applied by rewriting (3) as

$$
Z=\frac{X_{1} Y_{1}}{\hat{X}_{1}+Y_{1}}
$$

where $X_{1}=\left|c_{X}\right|^{2}, \hat{X}_{1}=\rho\left|\hat{c}_{X}\right|^{2}$, and $Y_{1}=\rho \beta^{2}$. A practical interpretation of this observation is summarized in the following Proposition.

Proposition 1: For medium and high SNRs and Rayleigh fading in the $S-R$ link, the VGR scheme with Gaussian estimation error at the relay (Scheme 2) is equivalent to the VGR scheme with outdated CSI at the relay (Scheme 1). However, when the relay gain for both schemes is set as in (1), Scheme 2 employs a (statistically) lower relay gain than Scheme 1. Consequently, the performance of Scheme 2 can be derived directly from the model used for Scheme 1, by applying a scaling factor $\rho$ in (1), where $\rho$ is given in (7); or equivalently, by replacing $\Omega_{Y}$ in Scheme 2 with $\rho \Omega_{Y}$.

Proof: For the case of Rayleigh fading in the $S-R$ link, $\hat{c}_{X}$ and $c_{X}$ are both zero-mean complex Gaussian RVs. The proof then follows directly from (3) and (8), by letting $E\left\langle X_{1}\right\rangle=E\left\langle\hat{X}_{1}\right\rangle=\Omega_{X}$ and $E\left\langle Y_{1}\right\rangle=\rho \Omega_{Y}$, in conjunction with the fact that the correlation between $\hat{X}_{1}$ and $X_{1}$ is identical to the correlation between $\hat{X}$ and $X$ since $\hat{X}_{1}$ is a scaled version of $\hat{X}$.

\section{Performance Analysis}

The analysis presented below gives lower bounds for the $\mathrm{OP}$ and ABEP of the considered three-node VGR system. As demonstrated in Section VII, these bounds are highly accurate approximations for moderate and high transmit SNRs.

\section{A. Lower Bound on Outage Probability}

The considered system experiences an outage when the instantaneous SNR $\gamma$ at the receiver at $D$ drops below some predefined outage threshold $\gamma_{t h}$. The OP equals the CDF of $Z$ evaluated at $\delta=\gamma_{t h} / \Gamma_{0}$, i.e.,

$$
P_{\text {out }}=F_{\gamma}\left(\gamma_{t h}\right)=F_{Z}\left(\gamma_{t h} / \Gamma_{0}\right) .
$$

By following two different approaches, we derive two alternative expressions for the $\mathrm{CDF}$ of $Z$, referred to as $C D F$ form I and CDF form II. Although having different analytical forms, both expressions produce the same numerical value for a given set of input parameters. 
1) CDF form I: Based on straightforward probability theory, the CDF of $Z$ can be expressed as

$$
\begin{aligned}
\operatorname{Pr}\{Z \leq \delta\} & =\operatorname{Pr}\left\{Y \geq \frac{\delta \hat{X}}{X-\delta}, X \leq \delta\right\} \\
& +\operatorname{Pr}\left\{Y<\frac{\delta \hat{X}}{X-\delta}, X>\delta\right\} .
\end{aligned}
$$

By noting that $Y \geq \frac{\delta \hat{X}}{X-\delta}$ is always true when $X \leq \delta$, (10) yields $C D F$ form $I$

$F_{Z}(\delta)=F_{X}(\delta)+\int_{\hat{x}=0}^{\infty} \int_{x=\delta}^{\infty} F_{Y}\left(\frac{\delta \hat{x}}{x-\delta}\right) f_{X \hat{X}}(x, \hat{x}) d x d \hat{x}$

where $F_{Y}(\cdot)$ and $f_{X \hat{X}}(\cdot)$ are given by (5) and (6), respectively.

2) $C D F$ form II: Alternatively to (11), the CDF of $Z$ can be expressed as

$$
\begin{aligned}
\operatorname{Pr} & \{Z \leq \delta\}=\operatorname{Pr}\left\{X \leq \delta\left(1+\frac{\hat{X}}{Y}\right)\right\} \\
& =\int_{0}^{\infty} \int_{0}^{\infty} F_{X \mid \hat{X}}\left(\delta\left(1+\frac{\hat{x}}{y}\right) \mid \hat{x}\right) f_{\hat{X}}(\hat{x}) f_{Y}(y) d \hat{x} d y \\
& =\int_{0}^{\infty} \int_{0}^{\infty} \frac{\int_{0}^{\delta(1+\hat{x} / y)} f_{X \hat{X}}(x, \hat{x}) d x}{f_{\hat{X}}(\hat{x})} f_{\hat{X}}(\hat{x}) f_{Y}(y) d \hat{x} d y
\end{aligned}
$$

By differentiating (12) with respect to (w.r.t.) $\delta$ and after a simple change of variables, we obtain

$f_{Z}(\delta)=\int_{0}^{\infty} \int_{0}^{\infty} v(1+u) f_{X \hat{X}}(\delta(1+u), u v) f_{Y}(v) d v d u$.

Assuming $m_{Y}$ is a positive integer, after the application of (4) and (6) in (13), the integration w.r.t. variable $v$ is solved by utilizing [21, Eq. (3.15.2.9)], which yields

$$
\begin{aligned}
& f_{Z}(\delta)=(1-\rho)^{m_{Y}}\left(\frac{m_{X}}{\Omega_{X}}\right)^{m_{X}-m_{Y}}\left(\frac{m_{Y}}{\Omega_{Y}}\right)^{m_{Y}} \frac{m_{Y} \delta^{m_{X}-1}}{\Gamma\left(m_{X}\right)} \\
& \int_{0}^{\infty} \frac{u^{m_{X}-1}(u+1)^{m_{X}}}{[u+(1-\rho) \Delta]^{m_{X}+m_{Y}}} \exp \left(-\frac{m_{X} \delta}{\Omega_{X}} \frac{(u+\Delta)(u+1)}{u+(1-\rho) \Delta}\right) \\
& \times L_{m_{Y}}^{m_{X}-1}\left(-\frac{m_{X} \rho \delta}{(1-\rho) \Omega_{X}} \frac{u(u+1)}{u+(1-\rho) \Delta}\right) d u,
\end{aligned}
$$

where $\Delta=m_{Y} \Omega_{X} /\left(m_{X} \Omega_{Y}\right)$ and $L_{n}^{a}(\cdot)$ is a generalized Laguerre polynomial, defined by [22, Eq. (8.970.1)]. Applying the definition of the generalized Laguerre polynomial in (14), we obtain

$$
\begin{aligned}
& f_{Z}(\delta)=\frac{m_{Y}(1-\rho)^{m_{Y}}}{\Gamma\left(m_{X}\right)}\left(\frac{m_{X}}{\Omega_{X}}\right)^{m_{X}-m_{Y}} \\
& \times\left(\frac{m_{Y}}{\Omega_{Y}}\right)^{m_{Y}} \sum_{n=0}^{m_{Y}}\left(\begin{array}{c}
m_{X}+m_{Y}-1 \\
m_{Y}-n
\end{array}\right)\left(\frac{m_{X} \rho}{(1-\rho) \Omega_{X}}\right)^{n} \\
& \times \frac{\delta^{m_{X}+n-1}}{n !} \int_{0}^{\infty} \frac{u^{m_{X}+n-1}(u+1)^{m_{X}+n}}{[u+(1-\rho) \Delta]^{m_{X}+m_{Y}+n}} \\
& \times \exp \left(-\frac{m_{X} \delta}{\Omega_{X}} \frac{(u+\Delta)(u+1)}{u+(1-\rho) \Delta}\right) d u .
\end{aligned}
$$

Next, we integrate (15) w.r.t. $\delta$ and obtain the CDF form II,

$$
\begin{aligned}
& F_{Z}(\delta)=\frac{m_{Y}[(1-\rho) \Delta]^{m_{Y}}}{\Gamma\left(m_{X}\right)} \sum_{n=0}^{m_{Y}}\left(\begin{array}{c}
m_{X}+m_{Y}-1 \\
m_{Y}-n
\end{array}\right) \\
& \times \frac{1}{n !}\left(\frac{\rho}{1-\rho}\right)^{n} \int_{0}^{\infty} \frac{u^{m_{X}+n-1}}{(u+(1-\rho) \Delta)^{m_{Y}}(u+\Delta)^{m_{X}+n}} \\
& {\left[\Gamma\left(m_{X}+n\right)-\Gamma\left(m_{X}+n, \frac{m_{X} \delta}{\Omega_{X}} \frac{(u+\Delta)(u+1)}{u+(1-\rho) \Delta}\right)\right] d u .}
\end{aligned}
$$

Note that $C D F$ form II, given by (16), is simpler to use since it requires only a single numerical integration, whereas $C D F$ form I, given by (11), requires a double numerical integration. However, both CDFs are required later for the asymptotic performance analysis of the system under different fading conditions.

\section{B. Lower Bound on Average Error Probability}

We now derive a tight lower bound for the ABEP for those modulation formats whose conditional BEP has the analytical form $P_{E}(\gamma)=a E\langle Q(\sqrt{b \gamma})\rangle$, where $a>0$, $b>0$ and $Q(x)=\frac{1}{\sqrt{2 \pi}} \int_{x}^{\infty} e^{-t^{2} / 2} d t$ is the Gaussian $Q$ function. Depending on the choice of $a$ and $b$, the above analytical form is applicable for binary/quadrature phase shift keying (BPSK/QPSK), frequency shift keying (FSK), and $M$ ary quadrature amplitude modulation (M-QAM) and coherent demodulation [27]. Using the approach presented in [28], the ABEP is determined by

$$
\bar{P}_{E}=\frac{a}{\sqrt{2 \pi}} \int_{0}^{\infty} F_{Z}\left(\frac{t^{2}}{b \Gamma_{0}}\right) \exp \left(-\frac{t^{2}}{2}\right) d t .
$$

Introducing (16) into (17), we obtain the following expression for the ABEP lower bound,

$$
\begin{aligned}
& \bar{P}_{E}=\frac{a}{2}-\frac{a m_{Y}[(1-\rho) \Delta]^{m_{Y}}}{\sqrt{2 \pi} \Gamma\left(m_{X}\right)} \sqrt{\frac{b \Gamma_{0} \Omega_{X}}{m_{X}}} \\
& \times \sum_{n=0}^{m_{Y}}\left(\begin{array}{c}
m_{X}+m_{Y}-1 \\
m_{Y}-n
\end{array}\right) \frac{\Gamma\left(m_{X}+n+1 / 2\right)}{\Gamma(n+1)}\left(\frac{\rho}{1-\rho}\right)^{n} \\
& \times \int_{0}^{\infty} \frac{u^{m_{X}+n-1}(u+1)^{-1 / 2}}{[u+(1-\rho) \Delta]^{m_{Y}-1 / 2}(u+\Delta)^{m_{X}+n+1 / 2}} \\
& \times{ }_{2} F_{1}\left(\frac{1}{2}, \frac{1}{2}+m_{X}+n ; \frac{3}{2} ;-\frac{b \Omega_{X} \Gamma_{0}}{2 m_{X}} \frac{u+(1-\rho) \Delta}{(u+\Delta)(u+1)}\right) d u,
\end{aligned}
$$

where ${ }_{2} F_{1}(\cdot)$ is the Gaussian hypergeometric function defined in [25, Eq. (15.1.1)]. For a given set of system and channel parameters, (18) can be solved numerically by applying the Laguerre-Gauss quadrature rule [25, Eq. (25.4.45)].

In deriving (18), we have split (17) into two integrals, corresponding to the two terms in the square brackets of (16). The first integral equals $a / 2$, which is the minuend in (18). This closed-form solution is obtained by changing the orders of integration and summation, where the sum with respect to the summation index $n$ is solved by applying the binomial formula [25, Eq. (3.1.1)], and then the integral is solved in closed-form by applying [22, Eq. (3.194-3)]. The subtrahend 
of (18) is obtained by exploiting the following formula:

$$
\begin{aligned}
& \int_{0}^{\infty} \Gamma\left(m_{X}+n, c t^{2}\right) \exp \left(-\frac{t^{2}}{2}\right) d t \\
& =\frac{\Gamma\left(m_{X}+n+1 / 2\right)}{\sqrt{c}}{ }_{2} F_{1}\left(\frac{1}{2}, \frac{1}{2}+m_{X}+n ; \frac{3}{2} ;-\frac{1}{2 c}\right),
\end{aligned}
$$

which is obtained by successively applying [22, Eq. (6.4551)], [25, Eq. (15.3.4)], and [25, Eq. (15.3.3)].

\section{PAPR ANALYSIS}

For perfect CSI, the main advantage of the AF relay gain shown in (1) is that it ensures a constant relay transmit power ${ }^{5}$. When the CSI at the relay is outdated, however, its ability to invert the attenuation caused by the $S-R$ link is impaired, resulting in larger fluctuations of the relay transmit power. Below, we quantify these fluctuations of the relay transmit power through the well-known crest factor, which is also known as the PAPR [32], [33]. The PAPR, $U$, is defined as $U \triangleq P_{R} /\left(E\left\langle P_{R}\right\rangle\right)$, where $P_{R}$ is the relay's transmit power for given $G$, and $E\left\langle P_{R}\right\rangle$ is the mean transmit power of the relay. Hence, the relay's ability to maintain constant transmit power is reflected in the statistics of the PAPR. The cases of VGR and FGR are considered separately.

\section{A. $V G R$}

It follows from (1) that the relay output power is given by

$P_{R}=G^{2}\left(E_{S} \alpha^{2}+N_{0}\right)=E_{R} \frac{E_{S} \alpha^{2}+N_{0}}{E_{S} \hat{\alpha}^{2}+N_{0}}=E_{R} \frac{X+1 / \Gamma_{0}}{\hat{X}+1 / \Gamma_{0}}$.

Notice from (20) that the relay's average transmit power is not the same as that of VGR with perfect CSI, and is actually a function of $\rho$. Denoting the relay's average transmit power by $\bar{E}_{R}^{V G R},(20)$ in conjunction with (6) and [21, Eq. (2.15.3/2)], yields

$$
\begin{aligned}
& \bar{E}_{R}^{V G R}=E\left\langle P_{R}\right\rangle \\
& =E_{R} \int_{0}^{\infty} \int_{0}^{\infty} \frac{X+1 / \Gamma_{0}}{\hat{X}+1 / \Gamma_{0}} f_{X \hat{X}}(X, \hat{X}) d X d \hat{X}=E_{R} \Psi,
\end{aligned}
$$

where

$$
\begin{aligned}
& \Psi=1+(1-\rho) \\
& \times\left[m_{X} \exp \left(\frac{m_{X}}{\Gamma_{0} \Omega_{X}}\right) \epsilon_{m_{X}}\left(\frac{m_{X}}{\Gamma_{0} \Omega_{X}}\right)\left(1+\frac{1}{\Gamma_{0} \Omega_{X}}\right)-1\right],
\end{aligned}
$$

and $\epsilon_{n}(\cdot)$ denotes the exponential integral, defined as $\epsilon_{n}(z)=$ $\int_{1}^{\infty}\left(e^{-z t}\right) /\left(t^{n}\right) d t$ [25, Eq. (5.1.4)]. Interestingly, we observe that $\bar{E}_{R}^{V G R}=E_{R}$ for $\rho=1 ; \bar{E}_{R}^{V G R}>E_{R}$ for any $0 \leq \rho<1$. This implies that, when the relay gain in a VGR application with oudated CSI is set as if perfect CSI was available, the relay's average transmit power is higher than expected. This fact should be taken into account when the performance of

\footnotetext{
${ }^{5}$ In fact, the signal at the input of the relay experiences two types of fluctuations, caused by small-scale fading and noise. Here, by "constant" we refer to the absence of fluctuations caused by small-scale fading, since these fluctuations are compensated by the gain in (1).
}

VGR with outdated CSI is compared with that of other relevant schemes. The PAPR for VGR is obtained from (20) and (21) as

$$
U_{V G}=\frac{1}{\Psi} \frac{X+1 / \Gamma_{0}}{\hat{X}+1 / \Gamma_{0}} .
$$

It is worth noting here that (23) applies to both cases of outdated CSI and Gaussian estimation error. This observation follows directly from (23) and Proposition 1. For this reason, in the sequel we refer to the PAPR performance of the cases of VGR with outdated CSI and Gaussian estimation error simply as PAPR performance of VGR. It follows from Proposition 1 that $\bar{E}_{R}^{V G R}$ for the case of Gaussian estimation error is obtained from (21), by setting $m_{X}=1$ and applying a correction factor $\rho$.

A typical measure for quantifying the fluctuations in the relay transmit power is the CCDF of $U_{V G}$ [32]; that is, the probability that $U_{V G}$ exceeds a given value, denoted by $\varepsilon$. This is an important metric as it can be used to quantify the probability that a given power amplifier at the relay operates in its non-linear region, where signal distortion occurs [6]. From another perspective, schemes with high probability of the PAPR exceeding a given $\varepsilon$ would require an amplifier with an extended linear region, increasing its cost. Although an exact study of the CCDF of $U_{V G}$ is cumbersome, for sufficiently high SNR, the term $1 / \Gamma_{0}$ can be neglected in both the numerator and denominator of (23), yielding a high SNR approximation of $U_{V G}$, which is denoted here by $U_{V G}^{\prime}$, and given by $U_{V G}^{\prime}=\frac{1}{\Psi} \frac{X}{\hat{X}}$. The PDF of $U_{V G}^{\prime}$ is derived as

$$
\begin{aligned}
f_{U_{V G}^{\prime}}(u) & =\int_{0}^{\infty} \Psi f_{U_{V G}^{\prime}, \hat{X}}(\Psi u, \omega) d \omega \\
& =\Psi \int_{0}^{\infty} \omega f_{X, \hat{X}}(\Psi u \omega, \omega) d \omega .
\end{aligned}
$$

Plugging (6) into (24) and using [21, Eq. 2.15.3/2] yields

$$
\begin{aligned}
f_{U_{V G}^{\prime}}(u) & =\frac{2^{2 m_{X}-1}(1-\rho)^{m_{X}} \Gamma\left(m_{X}+\frac{1}{2}\right) \Psi}{\sqrt{\pi} \Gamma\left(m_{X}\right)} \\
& \times \frac{(\Psi u)^{m_{X}-1}(\Psi u+1)}{\left[\Psi^{2} u^{2}+2(1-2 \rho) \Psi u+1\right]^{m_{X}+\frac{1}{2}}} .
\end{aligned}
$$

The CCDF of $U_{V G}^{\prime}$ is obtained from (25) as

$$
\operatorname{Pr}\left\{U_{V G}^{\prime}>\varepsilon\right\}=\int_{\varepsilon}^{\infty} f_{U_{V G}^{\prime}}(u) d u .
$$

It is noted that for the values of interest, i.e., $\varepsilon>1$, (26) represents an upper bound on the CCDF of the exact PAPR, $U_{V G}$, since for any $\varepsilon>1, X>\hat{X}$ holds, hence $U_{V G}^{\prime}>U_{V G}$ holds as well. Numerical examples of the performance of VGR with outdated CSI, in terms of the CCDF of the PAPR at the relay output, are provided in Section VI.

\section{B. $F G R$}

There are two common variations of FGR: The so-called "semi-blind" FGR, which employs a relay gain which equals the average value of the gain employed by VGR [34], [35]; and the so-called average power scaling (APS) FGR, where the fixed gain is selected as in (1) with $\hat{\alpha}^{2}$ replaced by its mean, $\Omega_{X}$ [36]. The PAPR for these two variations of FGR is studied below. 
1) Semi-Blind FGR: The relay gain in semi-blind FGR is given by [34], [35]

$$
G^{2}=E\left\langle\frac{E_{R}}{E_{S} \hat{\alpha}^{2}+N_{0}}\right\rangle
$$

The relay transmit power is expressed as

$$
\begin{aligned}
P_{R} & =G^{2}\left(E_{S} \alpha^{2}+N_{0}\right) \\
& =E_{R}\left(X+1 / \Gamma_{0}\right) E\left\langle\frac{1}{\hat{X}+1 / \Gamma_{0}}\right\rangle .
\end{aligned}
$$

By averaging over the distributions of $\hat{X}$ and $X$, given in (4), we obtain $E\left\langle P_{R}\right\rangle$ as

$$
\begin{aligned}
E\left\langle P_{R}\right\rangle & =E_{R} m_{X} \exp \left(\frac{m_{X}}{\Gamma_{0} \Omega_{X}}\right) \\
& \times \epsilon_{m_{X}}\left(\frac{m_{X}}{\Gamma_{0} \Omega_{X}}\right)\left(1+\frac{1}{\Gamma_{0} \Omega_{X}}\right) .
\end{aligned}
$$

We notice from (29) that $E\left\langle P_{R}\right\rangle>E_{R}$, implying that the parameter $E_{R}$ used for the relay gain in the semi-blind FGR scenario does not correspond to the relay's average transmit power, but to an underestimation of it. This fact should be taken into account when semi-blind FGR is compared with other relevant schemes.

The PAPR for semi-blind FGR is obtained from (28) and (29) as

$$
U_{F G_{S B}}=\frac{X+1 / \Gamma_{0}}{\Omega_{X}+1 / \Gamma_{0}}
$$

Considering the fact that $X$ is Gamma distributed, the CCDF of $U_{F G_{S B}}$ is derived from (30) and (5) as

$$
\begin{aligned}
& \operatorname{Pr}\left\{U_{F G_{S B}}>\varepsilon\right\} \\
& =\frac{1}{\Gamma\left(m_{X}\right)} \Gamma\left(m_{X}, \frac{m_{X}\left[\varepsilon\left(\Omega_{X}+\frac{1}{\Gamma_{0}}\right)-\frac{1}{\Gamma_{0}}\right]}{\Omega_{X}}\right) .
\end{aligned}
$$

2) APS FGR: For the case of APS FGR, the relay gain is given as [36], [37], [38]

$$
G^{2}=\frac{E_{R}}{E_{S} E\left\langle\hat{\alpha}^{2}\right\rangle+N_{0}}
$$

leading to the following expression for the relay transmit power

$$
P_{R}=\frac{E_{R}}{E_{S} \Omega_{X}+N_{0}}\left(E_{S} \alpha^{2}+N_{0}\right) \text {. }
$$

It is easy to notice from (33) that $E\left\langle P_{R}\right\rangle=E_{R}$, i.e., the relay's average transmit power equals $E_{R}$. Consequently, the PAPR for APS FGR is also given by (30).

3) On the FGR Variations: Interesting observations can be made regarding the PAPR and the relay's average transmit power of semi-blind and APS FGR. First, we notice that the PAPR for APS FGR is exactly the same as that of semiblind FGR. This result is in accordance with the intuition that, whichever fixed gain value the relay applies, the instantaneous and the average transmit power will be scaled by the same coefficient, hence the PAPR will be the same irrespective of the gain. Second, semi-blind FGR results in a higher average relay transmit power than APS FGR, which leads to better performance, as noted in the literature (see, e.g., [38]). In fact,
TABLE I

AVERAGe Relay Transmit POWER FOR VGR WITH OUdATEd CSI, SEMI-BLIND FGR, AND APS FGR

$$
A=m_{X} \exp \left(\frac{m_{X}}{\Gamma_{0} \Omega_{X}}\right) \epsilon_{m_{X}}\left(\frac{m_{X}}{\Gamma_{0} \Omega_{X}}\right)\left(1+\frac{1}{\Gamma_{0} \Omega_{X}}\right)
$$

\begin{tabular}{|c|c|}
\hline Relaying Scheme & Average Relay Transmit Power \\
\hline VGR, Outdated CSI & $E_{R}[1+(1-\rho)(A-1)]$ \\
\hline VGR, Gaussian Estimation Error & $\rho E_{R}[1+(1-\rho)(A-1)]$ \\
\hline Semi-blind FGR & $E_{R} A$ \\
\hline APS FGR & $E_{R}$ \\
\hline
\end{tabular}

the gain in semi-blind FGR is normalized such that it equals the average gain of VGR; the gain in APS FGR is normalized such that the relay transmit power equals that of VGR. Hence if, for comparison purposes, the same relay transmit power is assumed for both cases, semi-blind FGR and APS FGR represent two different viewpoints of the same technique. For this reason, in the sequel, we use the term FGR to refer to both semi-blind and APS FGR.

\section{Average Relay Transmit Power}

For the reader's convenience, a summary of the results regarding the average relay transmit power for VGR with outdated CSI, VGR with Gaussian estimation error, semi-blind FGR, and APS FGR is provided in Table I.

\section{ASYMPtotic PERformance ANALYSIS AND POWER ALLOCATION}

The expressions derived Section III are not simple enough to provide insight into the performance of VGR with outdated CSI. To this end, below we concentrate on the asymptotic OP and ABEP, shedding some light on the system's asymptotic behavior when $\Gamma_{0} \rightarrow \infty$. These asymptotic expressions are then used for optimizing the power allocation of VGR with outdated CSI at the relay.

\section{A. Asymptotic Performance Analysis}

Next, we investigate the behavior of the CDF expressions derived in Section III-A at infinitesimally small arguments. Such assumption is equivalent to the high SNR assumption, since for some fixed $\gamma_{t h}, \Gamma \rightarrow \infty$ implies $\delta \rightarrow 0$.

1) Case $m_{X}>m_{Y}$ : For this case, the asymptotic analysis is based on the CDF form I, given by (11), which, by neglecting the summand $\delta$ that appears in the joint PDF $f_{X \hat{X}}(\cdot, \cdot)$, can be alternatively written for $\delta \rightarrow 0$ as

$$
F_{Z}(\delta) \approx F_{X}(\delta)+\int_{0}^{\infty} \int_{0}^{\infty} F_{Y}\left(\frac{\delta v}{u}\right) f_{X \hat{X}}(u, v) d u d v .
$$

Since $\delta \rightarrow 0$, for some fixed value of $v / u$, the CDF of $Y$ that appears in the integrand of (34) can be approximated by using only the first two terms of the power series expansion of the incomplete Gamma function $\Gamma(\cdot, \cdot)$ [25, Eq. (6.5.29)], as

$$
F_{Y}(t) \sim \frac{t^{m_{Y}}}{\Gamma\left(m_{Y}+1\right)}\left(\frac{m_{Y}}{\Omega_{Y}}\right)^{m_{Y}},
$$


as $t=\delta y / x \rightarrow 0$. Using the same approximation as in (35), the first summand of (34), $F_{X}(\delta)$, can be ignored because it decays as $\delta^{m_{X}}$, and thus has a steeper slope compared to the second summand. Applying (35) in the double integral in (34), we obtain

$$
\begin{aligned}
& F_{Z}(\delta) \sim \frac{\delta^{m_{Y}}}{1-\rho}\left(\frac{m_{Y}}{\Omega_{Y}}\right)^{m_{Y}}\left(\frac{m_{X}}{\Omega_{X}}\right)^{m_{X}+1} \frac{1}{\rho^{\left(m_{X}-1\right) / 2}} \\
& \times \frac{1}{\Gamma\left(m_{X}\right) \Gamma\left(1+m_{Y}\right)} \int_{0}^{\infty} \int_{0}^{\infty} u^{m_{Y}+\frac{m_{X}-1}{2}} v^{\frac{m_{X}-1}{2}-m_{Y}} \\
& \times \exp \left(-\frac{m_{X}(u+v)}{(1-\rho) \Omega_{X}}\right) I_{m_{X}-1}\left(\frac{2 m_{X} \sqrt{\rho u v}}{(1-\rho) \Omega_{X}}\right) d v d u
\end{aligned}
$$

as $\delta \rightarrow 0$. The inner integral in (36) is solved in closed form by applying [21, Eq. (3.15.2.9)], yielding

$$
\begin{aligned}
& \int_{0}^{\infty} u^{m_{Y}+\frac{m_{X}-1}{2}} \exp \left(-\frac{m_{X}(u+v)}{(1-\rho) \Omega_{X}}\right) \\
& \times I_{m_{X}-1}\left(\frac{2 m_{X} \sqrt{\rho u v}}{(1-\rho) \Omega_{X}}\right) d u \\
& =\Gamma\left(m_{Y}+1\right)(\rho v)^{\frac{m_{X}-1}{2}}\left(\frac{(1-\rho) \Omega_{X}}{m_{X}}\right)^{m_{Y}+1} \\
& \times \exp \left(-\frac{m_{X} v}{\Omega_{X}}\right) \times L_{m_{Y}}^{\left(m_{X}-1\right)}\left(-\frac{m_{X} \rho v}{(1-\rho) \Omega_{X}}\right),
\end{aligned}
$$

where $L_{n}^{(\alpha)}(\cdot)$ is the generalized Laguerre polynomial of degree $n$, defined in [25, Eq. (22.3.9)]. The remaining integral w.r.t. variable $v$ is solved in closed form by straightforward application of [21, Eq. (3.24.1.7)]. Thus, (36) is reduced to

$$
\begin{aligned}
F_{Z}(\delta) & \sim \frac{\delta^{m_{Y}} \Gamma\left(m_{X}-m_{Y}\right)}{\Gamma\left(m_{X}\right)}\left(\frac{\rho m_{Y}}{\Omega_{Y}}\right)^{m_{Y}} \\
& \times P_{m_{Y}}^{\left(-2 m_{Y},-m_{X}\right)}\left(1-\frac{2}{\rho}\right)
\end{aligned}
$$

as $\delta \rightarrow 0$, where $P_{n}^{(\alpha, \beta)}(\cdot)$ denotes the Jacobi polynomial, defined by [22, Eq. (8.960-1)]. Note that (38) can be expressed as a finite sum, and is valid when $m_{Y}$ is a positive integer.

The ideal channel estimation case is obtained by setting $\rho=1$ in (38), in which case we apply [29, Eq. (05.06.03.0003.01)]. Thus, in this case, (38) reduces to $F_{Z}(\delta) \sim\left(\delta m_{Y} / \Omega_{Y}\right)^{m_{Y}} / \Gamma\left(m_{Y}+1\right)$, which is a well known asymptotic expression [30, Eq. (4)].

Applying (38) in (17) and using [22, Eq. (3.461.2)], we obtain the asymptotic ABEP for the case $m_{X}>m_{Y}$, for high transmit SNRs, i.e., $\Gamma_{0} \rightarrow \infty$, as

$$
\begin{aligned}
\bar{P}_{E} & \sim\left(\frac{1}{\Gamma_{0}}\right)^{m_{Y}} \frac{a \Gamma\left(m_{X}-m_{Y}\right)\left(2 m_{Y}-1\right) ! !}{2 \Gamma\left(m_{X}\right)} \\
& \times\left(\frac{\rho m_{Y}}{b \Omega_{Y}}\right)^{m_{Y}} P_{m_{Y}}^{\left(-2 m_{Y},-m_{X}\right)}\left(1-\frac{2}{\rho}\right),
\end{aligned}
$$

where $(\cdot)$ !! denotes the double factorial of its argument.

2) Case $m_{X}<m_{Y}$ : In this case, the asymptotic analysis is based on the $C D F$ form II, given by (16). As $\delta \rightarrow 0$, the expression in the last line of (16) is approximated similar to (35), yielding $\Gamma\left(m_{X}+n\right)-\Gamma\left(m_{X}+n, t\right) \sim t^{m_{X}+n} /\left(m_{X}+n\right)$. Introducing this approximation into (16), we notice that $\delta$ has the smallest exponent for the summation index $n=0$ (i.e.,
$\delta^{m_{X}}$ ), whereas the rest of the summation terms decay with steeper slopes w.r.t. $\delta$. Thus,

$$
\begin{aligned}
F_{Z}(\delta) & \sim \frac{\Gamma\left(m_{X}+m_{Y}\right) \Delta^{m_{Y}}(1-\rho)^{m_{Y}}}{\Gamma\left(m_{X}\right) \Gamma\left(m_{X}+1\right) \Gamma\left(m_{Y}\right)}\left(\frac{\delta m_{X}}{\Omega_{X}}\right)^{m_{X}} \\
& \times \int_{0}^{\infty} \frac{u^{m_{X}-1}(1+u)^{m_{X}}}{(x+(1-\rho) \Delta)^{m_{X}+m_{Y}}} d u
\end{aligned}
$$

The above integral can be solved in closed form by using [22, Eq. (3.197-5)], valid when $m_{Y}$ is a positive integer. If $m_{X}$ is also a positive integer, we apply the binomial formula to the term $(1+x)^{m_{X}}$ in the integrand of (40) and use [22, Eq. (3.194-3)] and [25, Eq. (15.4.1)], yielding

$$
\begin{aligned}
F_{Z}(\delta) & \sim \frac{\delta^{m_{X}}}{\Gamma\left(m_{X}+1\right)}\left(\frac{m_{X}}{\Omega_{X}}\right)^{m_{X}} \\
& \times{ }_{2} F_{1}\left(-m_{X}, m_{X}, 1-m_{Y},(1-\rho) \Delta\right)
\end{aligned}
$$

as $\delta \rightarrow 0$, where $\Delta=m_{Y} \Omega_{X} /\left(m_{X} \Omega_{Y}\right)$.

The ideal channel estimation case at the relay is obtained by setting $\rho=1$ in (41), in which case we apply [29, Eq. (07.23.03.0001.01)]. Thus, (41) reduces to $F_{Z}(\delta) \sim$ $\left(\delta m_{X} / \Omega_{X}\right)^{m_{X}} / \Gamma\left(m_{X}+1\right)$, which is a well known asymptotic expression [30, Eq. (4)].

Applying (41) in (17) and using [22, Eq. (3.461.2)], we obtain the asymptotic ABEP for the case $m_{X}<m_{Y}$

$$
\begin{aligned}
\bar{P}_{E} & \sim\left(\frac{1}{\Gamma_{0}}\right)^{m_{X}} \frac{a\left(2 m_{X}-1\right) ! !}{2 \Gamma\left(m_{X}+1\right)}\left(\frac{m_{X}}{b \Omega_{X}}\right)^{m_{X}} \\
& \times_{2} F_{1}\left(-m_{X}, m_{X}, 1-m_{Y},(1-\rho) \Delta\right),
\end{aligned}
$$

which is valid for high transmit SNRs, i.e., $\Gamma_{0} \rightarrow \infty$.

3) Case $m_{X}=m_{Y}=m$ : In this case, the asymptotic analysis is based on the PDF of the CDF form II, given by (15). Using partial fraction decomposition, the argument of the exponential function that appears in the integrand of (15) is rewritten as

$$
-\frac{m \delta}{\Omega_{X}} u-\frac{m \delta}{\Omega_{X}}\left(1+\frac{\rho \Delta(u+1)}{u+(1-\rho) \Delta}\right) .
$$

The term in parenthesis in (43) is bounded as

$$
\begin{aligned}
\min \left\{1+\rho \Delta, \frac{1}{1-\rho}\right\} & \leq 1+\frac{\rho \Delta(u+1)}{u+(1-\rho) \Delta} \\
& \leq \max \left\{1+\rho \Delta, \frac{1}{1-\rho}\right\},
\end{aligned}
$$

which means that both the lower and upper bounds in (44) are independent from $\delta$, and, consequently, the second term in (43) diminishes as $\delta \rightarrow 0$. Thus, the exponential function in (15) can be approximated as $\exp \left(-m \delta u / \Omega_{X}\right)$, and the resulting integral is solved in closed form as

$$
\begin{aligned}
& \int_{0}^{\infty} \frac{u^{m+n-1}(u+1)^{m+n}}{[u+(1-\rho) \Delta]^{2 m+n}} \exp \left(-\frac{m \delta u}{\Omega_{X}}\right) d u \\
& =\sum_{k=0}^{m+n}\left(\begin{array}{c}
m+n \\
k
\end{array}\right) \int_{0}^{\infty} \frac{u^{m+n+k-1}}{[u+(1-\rho) \Delta]^{2 m+n}} e^{-\frac{m \delta u}{\Omega_{X}}} d u \\
& =\sum_{k=0}^{m+n}\left(\begin{array}{c}
m+n \\
k
\end{array}\right) \Gamma(m+n+k)\left[\frac{(1-\rho) \Omega_{X}}{\Omega_{Y}}\right]^{k-m} \\
& \times U\left(m+n+k, k-m+1 ; \frac{m(1-\rho) \delta}{\Omega_{Y}}\right)
\end{aligned}
$$


where we utilized the binomial formula and [21, Eq. (2.1.3.1)]. In ( 45), $U(a ; b ; z)$ is the Tricomi confluent hypergeometric function [25, Eq. (13.2.5)], which, as $\delta \rightarrow 0$, is approximated according to [25, Eqs. (13.5.6)-(13.5.9)], as

$$
U(a ; b ; z) \sim \begin{cases}\frac{\Gamma(b-1)}{\Gamma(a)} z^{1-b}, & b>1, \\ -\frac{1}{\Gamma(a)}[\log (z)+\psi(a)+2 \mu], & b=1, \\ \frac{\Gamma(1-b)}{\Gamma(1+a-b)}, & b<1,\end{cases}
$$

where $\psi(\cdot)$ is the digamma function, defined in [25, Eq. (6.3.1)], and $\mu$ is Euler's constant.

Using (45) in (15), we obtain a double sum, where the index of the first sum, $n$, runs from 0 to $m$ and the index of the second sum, $k$, runs from 0 to $m+n$. At first glance, one could consider neglecting the summation terms for values of $n$ from 1 to $m$, because they decay as $\delta^{m+n-1}$ whereas the remaining term (for $n=0$ ) decays as $\delta^{m-1}$. However, considering the approximation (46) for $b>1$, we notice that, for $n>0$ and values of $k$ between $m+1$ and $m+n$, parameter $\delta$ appears as $\delta^{m+n-2}, \delta^{m+n-3}, \ldots, \delta^{m-1}$. Thus, the smallest exponent of $\delta$ appears for $k=m+n$, and, for each $n>0$, a single term of the other sum should be kept in the approximation $(k=m+n)$. Consequently, (15) is approximated for $\delta \rightarrow 0$ as

$$
\begin{aligned}
& f_{Z}(\delta) \sim \frac{m \delta^{m-1}}{\Gamma(m)}\left(\frac{(1-\rho) m}{\Omega_{Y}}\right)^{m} \\
& \times\left\{\left(\begin{array}{c}
2 m-1 \\
m
\end{array}\right) \sum_{k=0}^{m}\left(\begin{array}{c}
m \\
k
\end{array}\right) \Gamma(m+k)\left[\frac{(1-\rho) \Omega_{X}}{\Omega_{Y}}\right]^{k-m}\right. \\
& \times U\left(m+k, k-m+1 ; \frac{(1-\rho) m \delta}{\Omega_{Y}}\right) \\
& \left.+\sum_{n=1}^{m}\left(\begin{array}{c}
2 m-1 \\
m-n
\end{array}\right) \frac{1}{n}\left(\frac{\rho}{1-\rho}\right)^{n}\right\}
\end{aligned}
$$

By noting that the second argument of the Tricomi functions in (47) is smaller than or equal to one, we apply (46) in (47) for the cases $b=1$ (i.e., $k=m$ ) and $b<1$ (i.e., $k=0, \ldots, m-1$ ) to obtain the asymptotic CDF of $Z$ as

$$
\begin{aligned}
& F_{Z}(\delta) \sim \frac{\delta^{m}}{\Gamma(m)}\left(\frac{(1-\rho) m}{\Omega_{Y}}\right)^{m} \\
& \times\left\{( \begin{array} { c } 
{ 2 m - 1 } \\
{ m }
\end{array} ) \left[\frac{1}{m}+\log \left(\frac{\Omega_{Y}}{(1-\rho) m \delta}\right)-\psi(2 m)-2 \mu\right.\right. \\
& \left.+\sum_{k=0}^{m-1}\left(\begin{array}{c}
m \\
k
\end{array}\right) \frac{\Gamma(m+k) \Gamma(m-k)}{\Gamma(2 m)}\left(\frac{\Omega_{Y}}{(1-\rho) \Omega_{X}}\right)^{m-k}\right] \\
& \left.+\sum_{n=1}^{m}\left(\begin{array}{c}
2 m-1 \\
m-n
\end{array}\right) \frac{1}{n}\left(\frac{\rho}{1-\rho}\right)^{n}\right\}
\end{aligned}
$$

The case of perfect CSI is obtained by setting $\rho=1$ in (48), in which case we keep only a single term in each of the summations ( $k=0$ and $n=m$ ) and apply the known limit $\lim _{u \rightarrow 0} u^{m} \log (u)=0$. Thus, (48) reduces to

$$
F_{Z}(\delta) \sim \frac{\delta^{m}}{\Gamma(m+1)}\left[\left(\frac{m}{\Omega_{X}}\right)^{m}+\left(\frac{m}{\Omega_{Y}}\right)^{m}\right],
$$

which is a well known asymptotic expression [30, Eq. (4)]. The case of Rayleigh fading is obtained by setting $m=1$ in
(48), yielding the asymptotic CDF of $Z$

$F_{Z}(\delta) \sim \delta\left(\frac{1}{\Omega_{X}}+\frac{\rho}{\Omega_{Y}}\right)+\frac{\delta(1-\rho)}{\Omega_{Y}}\left[\log \left(\frac{\Omega_{Y}}{\delta(1-\rho)}\right)-\mu\right]$.

Applying (48) in (17) and using [22, Eqs. (3.381.4) and (4.352.1)], we obtain the asymptotic ABEP for the case $m_{X}=$ $m_{Y}=m$, as

$$
\begin{aligned}
& \bar{P}_{E} \sim\left(\frac{1}{\Gamma_{0}}\right)^{m} \frac{a(2 m-1) ! !}{2 \Gamma(m)}\left(\frac{(1-\rho) m}{b \Omega_{Y}}\right)^{m}\left\{\left(\begin{array}{c}
2 m-1 \\
m
\end{array}\right)\right. \\
& \times\left[\frac{1}{m}+\log \left(\frac{b \Omega_{Y} \Gamma_{0}}{2 m(1-\rho)}\right)-\psi\left(m+\frac{1}{2}\right)-\psi(2 m)\right. \\
& \left.-2 \mu+\sum_{k=0}^{m-1}\left(\begin{array}{c}
m \\
k
\end{array}\right) \frac{\Gamma(m+k) \Gamma(m-k)}{\Gamma(2 m)}\left(\frac{\Omega_{Y}}{(1-\rho) \Omega_{X}}\right)^{m-k}\right] \\
& \left.+\sum_{n=1}^{m}\left(\begin{array}{c}
2 m-1 \\
m-n
\end{array}\right) \frac{1}{n}\left(\frac{\rho}{1-\rho}\right)^{n}\right\}
\end{aligned}
$$

which is valid for high transmit SNRs, i.e., $\Gamma_{0} \rightarrow \infty$.

\section{B. Power Allocation}

Here, we propose a simple power allocation strategy that minimizes the OP of dual hop AF relaying with outdated CSI, under constraints on the total transmitted power. For the sake of simplicity and analytical tractability, the power allocation is based on the asymptotic expressions derived in Section V-A. Such approach follows other works on optimal power allocation for AF relaying with perfect CSI, such as [39], [40], and [41], which are also based on asymptotic outage probabilities. It is emphasized, however, that the effect of optimal power allocation in our scheme is different than that in these works. This is because, as opposed to the case of perfect CSI, imperfect CSI at the relay causes imperfect inversion of the $S-R$ channel which renders the power allocation disproportionate to the conditions of the $S-R$ and $R-D$ links. It is further noted that since the derived approximations are tight in the moderate and high SNR regime, as illustrated in Section VI, the proposed approach yields a tight approximation of the optimal power allocation in the aforementioned SNR region. Below, we focus on the case of $m_{X}=m_{Y}=m$; a similar analysis can be conducted for the cases of $m_{X}>m_{Y}$ and $m_{X}<m_{Y}$.

Our goal is to allocate the source transmit power, $E_{S}=E_{S}^{*}$, and the relay's average transmit power, $\bar{E}_{R}^{V G R}=E_{R}^{*}$, so as to minimize the asymptotic OP given by (48). The constraint in our optimization problem is on the total power budget $E_{T}$, i.e., $E_{S}+\bar{E}_{R}^{V G R}=E_{T}$, with the second summand given by (21). Note that further constraints can also be imposed (such as individual transmit power constraints for the source and the relay), but this would obscure the analysis of the achievable performance improvements.

We use variable $a$ to parameterize the transmit powers of the source $S$ and the relay $R$ w.r.t. the total power budget $E_{T}$, such that $E_{S}=a E_{T}$ and $\bar{E}_{R}^{V G R}=E_{R} \Psi=(1-a) E_{T}$. Introducing these terms into (2) and (1), the instantaneous SNR at the input of destination $D$ is given by

$$
\gamma=\Gamma_{T} \frac{X_{2} Y_{2}}{\hat{X}_{2}+Y_{2}}
$$


where $X_{2}=a \alpha^{2}, \hat{X}_{2}=a \hat{\alpha}^{2}, Y_{2}=(1-a) \beta^{2} / \Psi$, and $\Gamma_{T}=E_{T} / N_{0}$ is denoted as the total transmit SNR. Therefore, $E\left\langle X_{2}^{2}\right\rangle=E\left\langle\hat{X}_{2}^{2}\right\rangle=a \Omega_{X}$ and $E\left\langle Y_{2}^{2}\right\rangle=(1-a) \Omega_{Y} / \Psi$.

Thus, the objective function is the asymptotic OP of the considered system, $P_{\text {out }}$, which is given by (48) with $\delta$ replaced by $\gamma_{t h} / \Gamma_{T}, \Omega_{X}$ replaced by $a \Omega_{X}$, and $\Omega_{Y}$ replaced by $(1-a) \Omega_{Y} / \Psi$. We need to find the optimal value of $a=a_{0}$ that minimizes $P_{\text {out }}$ subject to the condition $0<a_{0}<1$.

1) Case $m \geq 2$ : An analytical expression for the exact $a_{0}$ is cumbersome to derive, since $\Psi$ depends on $E_{T}$ according to (21). Still, for the case $m \geq 2$, there is a relatively simple solution yielding close to the optimal $a_{0}$. Based on [25, Eq. (5.1.19)], $\Psi$ can be bounded as

$$
1<\Psi \leq \frac{m-\rho}{m-1}
$$

where the upper bound is tight for high transmit SNRs.

We can determine $a_{0}$ for minimization of $P_{\text {out }}$ when $\Psi$ is selected according to the upper bound of (53), i.e., $\Psi=\Psi_{0}=(m-\rho) /(m-1)$. It can be shown that the second derivative of $P_{\text {out }}$ w.r.t. variable $a$ is always larger or equal to zero, indicating that $P_{\text {out }}$ is a convex function w.r.t. variable $a$, and has a unique minimum value provided $a$ is in the range between 0 and 1 . The value $a=a_{0}$ that minimizes $P_{\text {out }}$ is obtained by setting the first derivative of the objective function w.r.t. variable $a$ equal to 0 , yielding the transcendental equation,

$$
\begin{aligned}
& \log \left[\frac{\left(1-a_{0}\right) \Omega_{Y} \Gamma_{T}}{\gamma_{t h}(1-\rho) m \Psi_{0}}\right]+\sum_{k=0}^{m-1}\left(\begin{array}{c}
m \\
k
\end{array}\right) \frac{\Gamma(m+k) \Gamma(m-k)}{\Gamma(2 m)} \\
& \times\left(\frac{1-a_{0}}{a_{0}} \frac{\Omega_{Y}}{(1-\rho) \Omega_{X} \Psi_{0}}\right)^{m-k}\left(1-\frac{1}{a_{0}}+\frac{k}{m a_{0}}\right) \\
& =\psi(2 m)+2 \mu-\frac{m[\Gamma(m)]^{2}}{\Gamma(2 m)} \sum_{k=1}^{m}\left(\begin{array}{c}
2 m-1 \\
m-k
\end{array}\right) \frac{1}{k}\left(\frac{\rho}{1-\rho}\right)^{k} .
\end{aligned}
$$

Then, the source transmit power and the relay's average transmit power are obtained as $E_{S}^{*}=a_{0} E_{T}$ and $E_{R}^{*}=$ $\left(1-a_{0}\right) E_{T}$, respectively. These values are close-to-optimal for high transmit SNRs.

Note that the above approach can also be applied for moderate transmit SNRs, because $a_{0}$ obtained from (54) assures that the power constraint, $E_{S}+\bar{E}_{R}^{V G R} \leq E_{T}$, is certainly met. Here, $\bar{E}_{R}^{V G R}$ is calculated from (21) with $\Gamma_{0}$ replaced by $a_{0} \Gamma_{T}$.

We now multiply both sides of (54) by $(1-\rho)^{m}$ and let $\rho \rightarrow$ 1 in order to obtain the perfect CSI case. In this case, the lower and the upper bounds of (53) coincide, and a simple closedform solution for the optimal power allocation is obtained as

$$
\frac{1}{a_{0 p}}=1+\left(\frac{\Omega_{X}}{\Omega_{Y}}\right)^{\frac{m}{1+m}},
$$

which, to the best of the authors' knowledge, is a novel result. In the case of perfect CSI under Rayleigh fading, (55) coincides with [39, Eq. (8)].

2) Case $m=1$ : For the Rayleigh case, based on [25, Eq. (5.1.19)], $\Psi$ is bounded as $1<\Psi \leq 1+(1-\rho) \Gamma_{0} \Omega_{X}$. Since $\Gamma_{0}=a \Gamma_{T}$, the upper bound of $\Psi$ depends on $a$ and $E_{T}$, thus, we cannot apply a similar approach as in the case

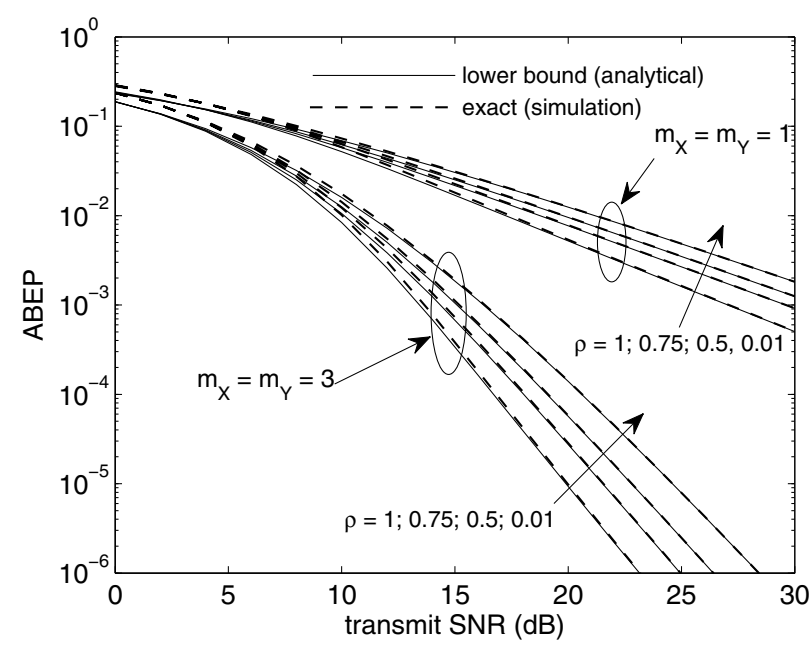

Fig. 1. ABEP for BPSK modulation vs. transmit SNR, when $\Omega_{X}=\Omega_{Y}=1$ (ideal CSI curve, $\rho=1$, is obtained by using [24, Eq. (9)]). Case of outdated CSI.

$m \geq 2$. Instead, it is possible to apply some suitable convex optimization method to determine the $a_{0}$ that minimizes $P_{\text {out }}$ [42]. These methods are readily implemented in standard computation software tools, such as Mathematica.

\section{NumericAl EXAMPLES AND Discussion}

Fig. 1 depicts the lower bound of the average ABEP for BPSK modulation of VGR with outdated CSI versus the transmit SNR, along with the results obtained through Monte Carlo simulations. Symmetric $S-R$ and $R-D$ channels (i.e., $\Omega_{X}=\Omega_{Y}$ ) and identical Nakagami- $m$ fading parameters for both links ( $m_{X}=m_{Y}=1$ and $\left.m_{X}=m_{Y}=3\right)$ are assumed. The lower ABEP bounds (full lines) were generated using (18), except for the ideal CSI case of $\rho=1$ where [24, Eq. (9)] was used. One observes a close correspondence between simulations and the ABEP bound, particularly for medium and high transmit SNRs, which validates our SNR approximation in (3). It is observed also that outdated CSI at the relay ( $\rho$ smaller than one) introduces a considerable performance degradation. However, such degradation is reflected only in a shift of the ABEP curves (i.e., loss in SNR), yet without affecting their asymptotic slopes.

The case of imperfect CSI at the relay with Gaussian estimation error is considered in Fig. 2. We observe a similar ABEP performance as in the outdated CSI case, which is expected since the model for the Gaussian estimation error case is practically another viewpoint of the model that describes the outdated CSI case (see Section II-B). The accuracy of the analytical lower bounds for the ABEP is confirmed by simulations. For Gaussian estimation errors, for the extreme case where $\sigma_{e}^{2}$ is large compared to $\Omega_{X}$, the ABEP performance is severely degraded. This result follows from (7) and Proposition 1: Large values of $\sigma_{e}^{2}$ result in small values of $\rho$, which in turn result in a severe degradation of the average $G$ value. Note that this observation is in contrast to the outdated CSI case, where the average value of $G$ is independent of $\rho$, hence the ABEP dependence on $\rho$ is relatively low. 


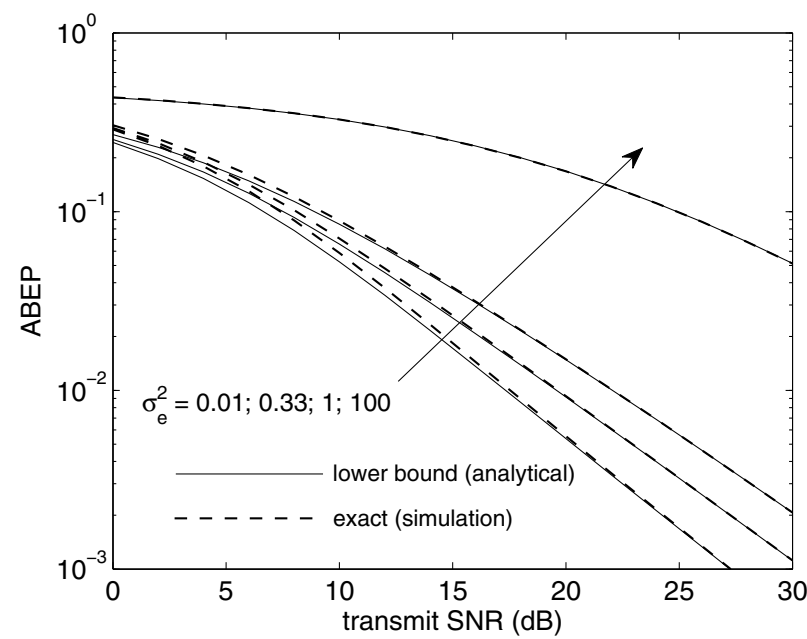

Fig. 2. ABEP for BPSK modulation vs. transmit SNR, when $\Omega_{X}=\Omega_{Y}=1$ (ideal CSI curve, $\rho=1$, is obtained by using [24, Eq. (9)]). Case of Gaussian estimation error, where the variance of the estimation error is denoted by $\sigma_{e}^{2}$, and $m_{X}=m_{Y}=1$.

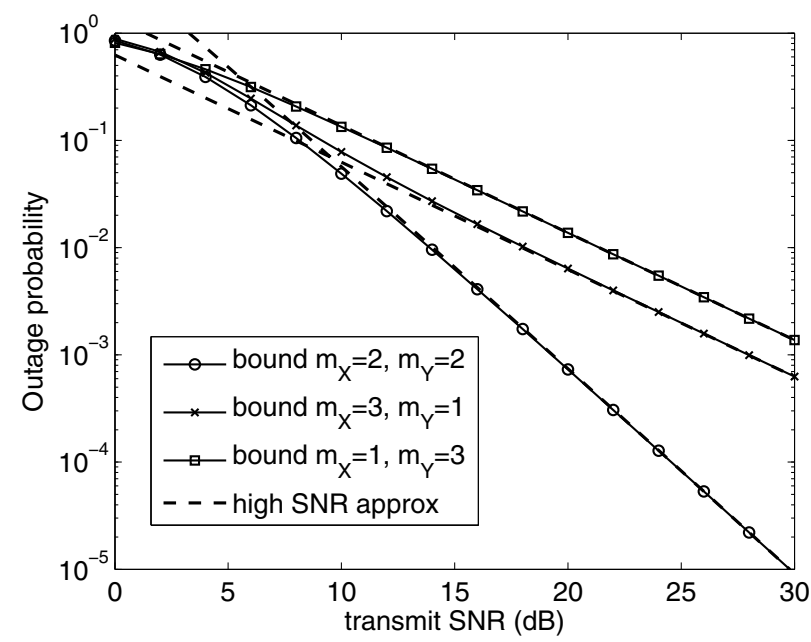

Fig. 3. Asymptotic OP and exact OP vs. $\Gamma_{0}$, when $\Omega_{X}=1, \Omega_{Y}=2$, $\rho=0.5$, and $\gamma_{t h}=1$.

In a similar context, Fig. 3 illustrates the outage performance of VGR, for $\Omega_{X}=1, \Omega_{Y}=2, \rho=0.5$, several combinations of $m_{X}$ and $m_{Y}$, and outage threshold $\gamma_{t h}=1$. Both the upper performance bounds and high SNR approximations, derived in Sections III and V-A, respectively, are presented, showing a close match for sufficiently high SNR. The combinations of $m_{X}$ and $m_{Y}$ were chosen such that $m_{X}+m_{Y}=4$, i.e., $m_{X}=1, m_{Y}=3 ; m_{X}=3, m_{Y}=1 ; m_{X}=2, m_{Y}=2$. We notice that the case of $m_{X}=3, m_{Y}=1$ outperforms that of $m_{X}=1, m_{Y}=3$, which is due to the fact that the overall performance is dominated by the bottleneck of the $S-R$ and $R-D$ links, hence the imperfect compensation of the $S-R$ link at the relay, in conjunction with $\Omega_{X}<\Omega_{Y}$, renders the $S-R$ link the bottleneck channel.

A comparison between VGR with outdated CSI and FGR, in terms of the ABEP, is presented in Fig. 4. In particular, the ABEPs for VGR and FGR are plotted versus the value of $\rho$. For a fair comparison, using Table I, the $E_{R}$ values used

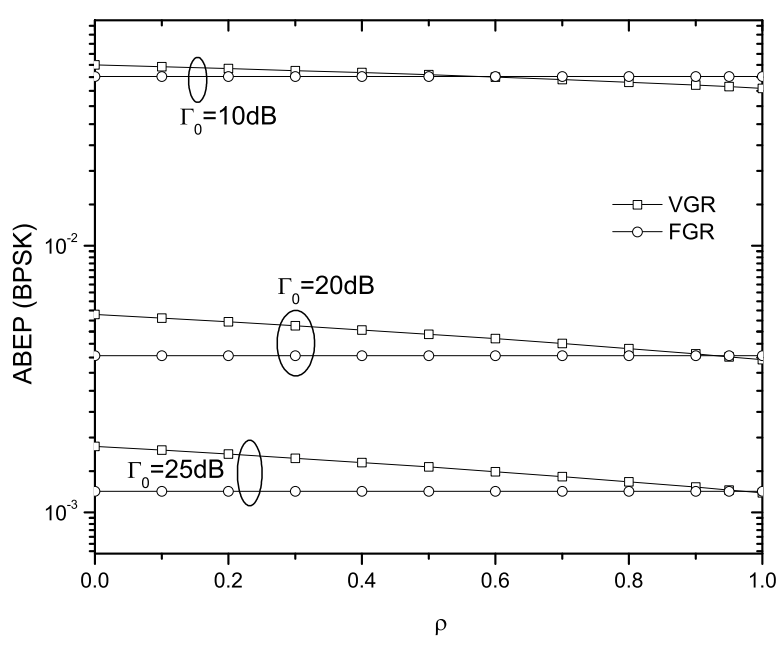

Fig. 4. ABEP (BPSK) of VGR with outdated CSI and FGR vs. the correlation coefficient $\rho$, assuming $m_{X}=3, m_{Y}=1$, and $\Omega_{X}=\Omega_{Y}=1$. For fair comparison, the relay gains for VGR and FGR were normalized such that the relay transmit SNR equals the source transmit SNR $\Gamma_{0}$ in both cases.

for VGR with outdated CSI and FGR were normalized such that the relay transmit power is the same for both schemes. The ABEP for FGR, which is by definition independent of $\rho$, was obtained from [4, Eq. (11)]; we note that the same curves for FGR are obtained from [34, Eq. (10)], in conjunction with [35, Eq. (12)], by dividing the parameter $\bar{\gamma}_{2}$ in [34, Eq. (10)] by $A$, where $A$ is given in Table I. It is observed that there exists a threshold value such that for values of $\rho$ smaller than this threshold VGR leads to inferior performance compared to FGR. The higher the value of the transmit SNR, $\Gamma_{0}$, the larger this threshold value for $\rho$.

For illustrating a more practical aspect of the comparison between VGR and FGR in terms of ABEP, let us consider the case where a source terminal moves at the speed of $50 \mathrm{~km} / \mathrm{h}$ and transmits at a carrier frequency of $2.4 \mathrm{GHz}$, resulting in a maximum Doppler frequency of $f_{d} \approx 100 \mathrm{~Hz}$. The corresponding correlation coefficient between $X$ and $\hat{X}$ is obtained via Jakes' model [31] as $\rho=J_{0}^{2}\left(2 \pi f_{d} T\right)$, where $f_{d}$ is the maximum Doppler frequency, $T$ is the time interval between consecutive estimation updates, and $J_{0}(\cdot)$ denotes the zeroth order Bessel function of the first kind [22, Eq. (8.411)]. For the above scenario, Fig. 5 illustrates the ABEP of VGR versus $T$, manifesting a considerable performance degradation for relatively high values of $T$. In Fig. 5 the ABEP performance of FGR and the direct communication link between source and destination (where no relaying takes place) is also presented. For a fair comparison between VGR anf FGR, the relay transmit power of FGR is set equal to that of VGR with outdated CSI. It is shown that, in order for VGR to outperform FGR, the CSI has to be updated at least every $1.35 \mathrm{msec}$, for transmit SNRs of $20 \mathrm{~dB}$ and $25 \mathrm{~dB}$.

Figs. 6 and 7 portray the performance improvements in VGR with outdated CSI when the available power budget $E_{T}$ (or, equivalently $\Gamma_{T}$ ) is optimally allocated between the source and the relay. We assume the system operates under Nakagami- $m$ fading with $m_{X}=m_{Y}=2$, and the optimal powers are allocated according to the transcendental equation 


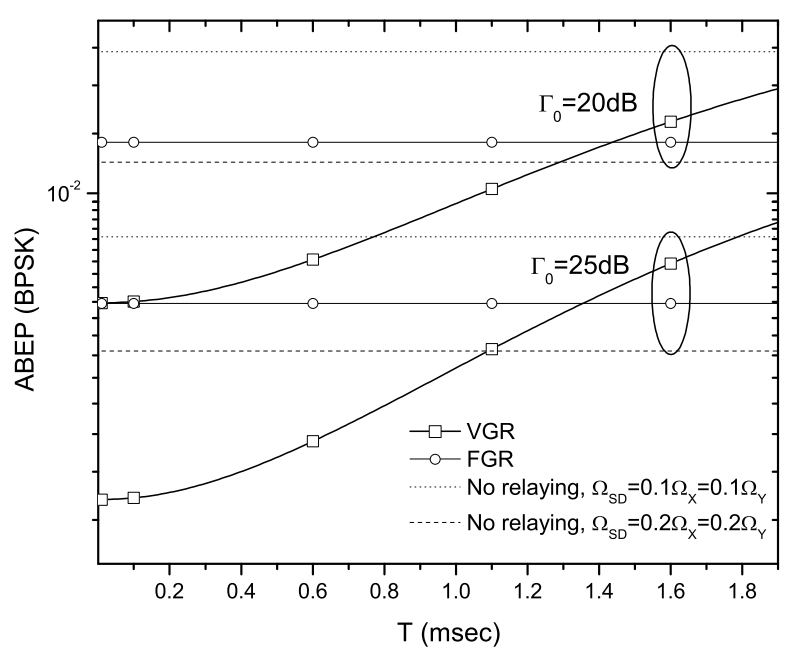

Fig. 5. ABEP (BPSK) vs. the update time interval of the estimation process, assuming a moving source at a speed of $50 \mathrm{~km} / \mathrm{h}$, a carrier frequency of 2.4 $\mathrm{GHz}, m_{X}=1, m_{Y}=1, \Omega_{X}=\Omega_{Y}=1$, for VGR and FGR. For fair comparison, the relay gains for VGR and FGR were normalized such that the relay transmit SNR is equal to the source transmit SNR $\Gamma_{0}$ in both cases.

(54), valid for the upper bound (53) but sufficiently tight for high transmit SNRs. Three different channel scenarios are considered: statistically equal $S-R$ and $R-D$ links $\left(\Omega_{X}=\Omega_{Y}=1\right)$, statistically weaker $S-R$ link $\left(\Omega_{X}=1\right.$ and $\left.\Omega_{Y}=10\right)$, and statistically stronger $S-R$ link $\left(\Omega_{X}=10\right.$ and $\Omega_{Y}=1$ ).

For these scenarios, Fig. 6 depicts the impact of $\rho$ on the system's OP. For decreasing $\rho$, we can clearly see that the influence of CSI imperfection is significant, and leads to some degradation of the OP performance. Compared to the case of equal power allocation $\left(a_{0}=0.5\right)$, the OP improvement with optimal power allocation is much more pronounced when the $S-R$ link is stronger than the $R-D$ link. Fig. 7 depicts the influence of the total available power budget on the OP performance of the considered system for optimal and equal power allocation, respectively, when $\rho=0.7$. The case of perfect CSI and optimal power allocation (dotted lines) is obtained using (55) and serves as a reference, where the curves for statistically asymmetric links coincide due to the symmetry of (55).

Fig. 7 portrays the significant performance degradation when the CSI is outdated. Compared to equal power allocation, we again notice a significant power gain (of several $\mathrm{dBs}$ ) for optimal power allocation when the $S-R$ link is statistically ten times stronger then the $R$ - $D$ link. If both links are statistically equal or the $R-D$ link is stronger, the system's performance does not benefit as much from optimal power allocation. The general conclusion from Fig. 7 is that when the CSI at the relay is outdated, the effect of power allocation is different than in the case of perfect CSI. In particular, it is noticed that since the source-relay channel is not perfectly compensated by the relay gain when the CSI at the relay is outdated, the performance improvement due to power allocation is higher for the case when the $S$ - $R$ channel is stronger than the $R-D$ channel, compared to the case when the $S-R$ channel is weaker than the $R-D$ channel.

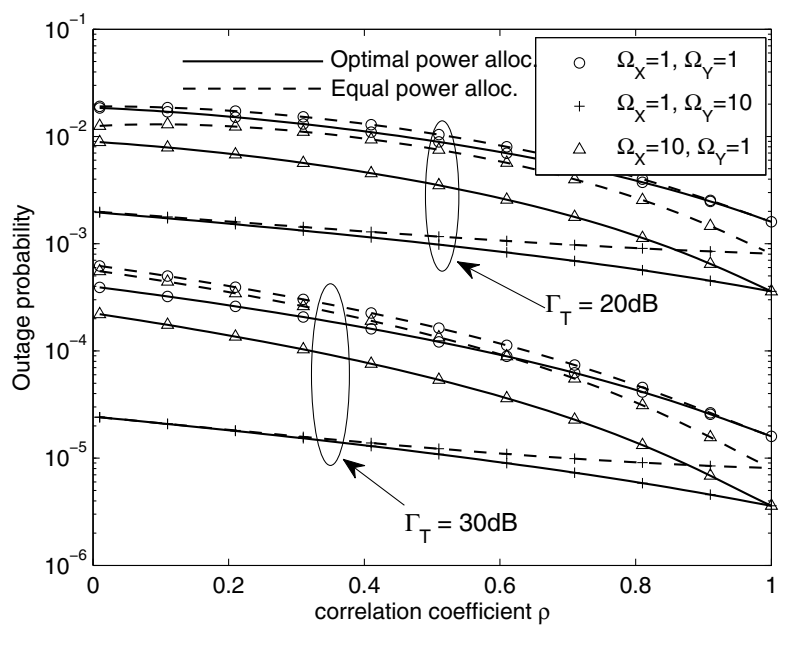

Fig. 6. Impact of correlation coefficient on the outage probability for optimal and equal power allocation in Nakagami- $m$ fading $\left(m_{X}=m_{Y}=2\right) . \rho=1$ denotes the case of perfect channel estimates. The total transmit budget is fixed to $\Gamma_{T}$, and $\gamma_{t h}=1$.

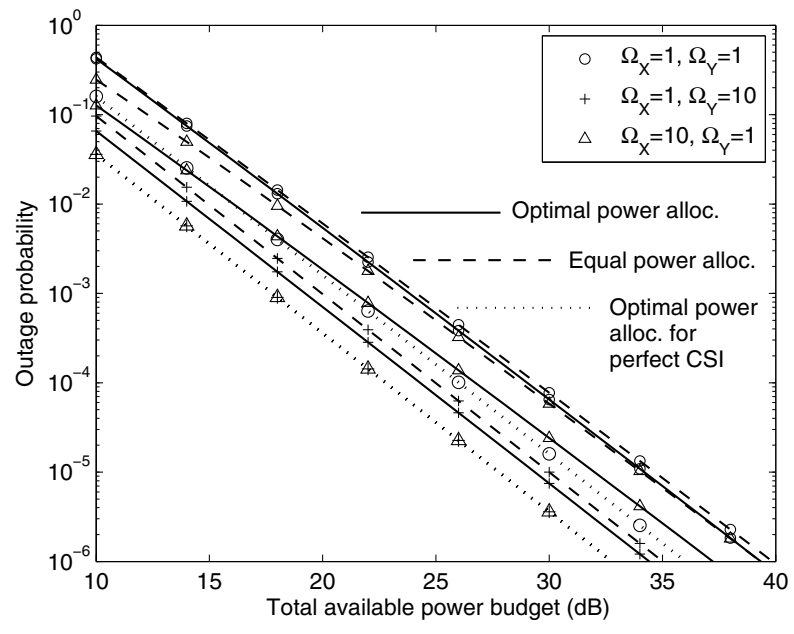

Fig. 7. Outage probability (OP) vs. total transmit budget for optimal and equal power allocation with outdated $(\rho=0.7)$ and perfect channel estimates $(\rho=1)$ in Nakagami- $m$ fading $\left(m_{X}=m_{Y}=2\right)$, and $\gamma_{t h}=1$.

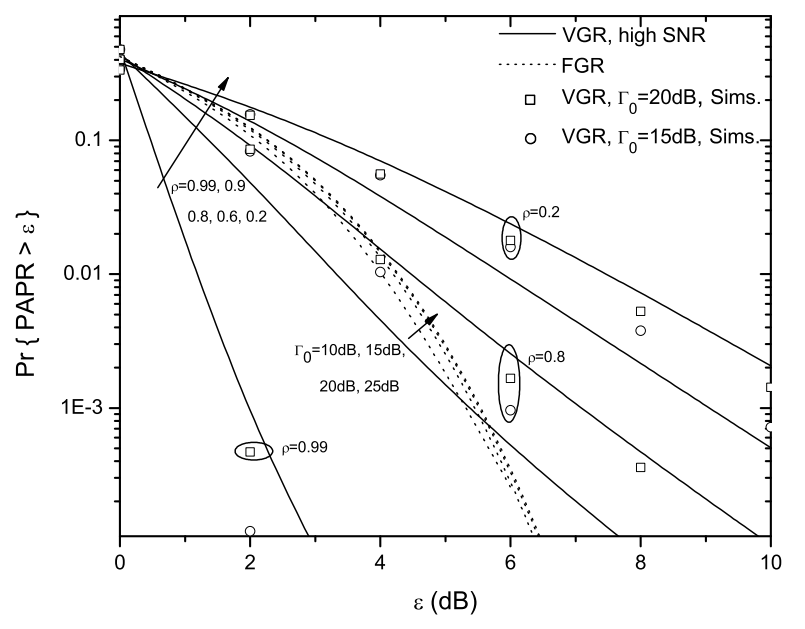

Fig. 8. CCDF of the PAPR for VGR with outdated CSI and FGR, for $m_{X}=3$, and $\Omega_{X}=1$. 


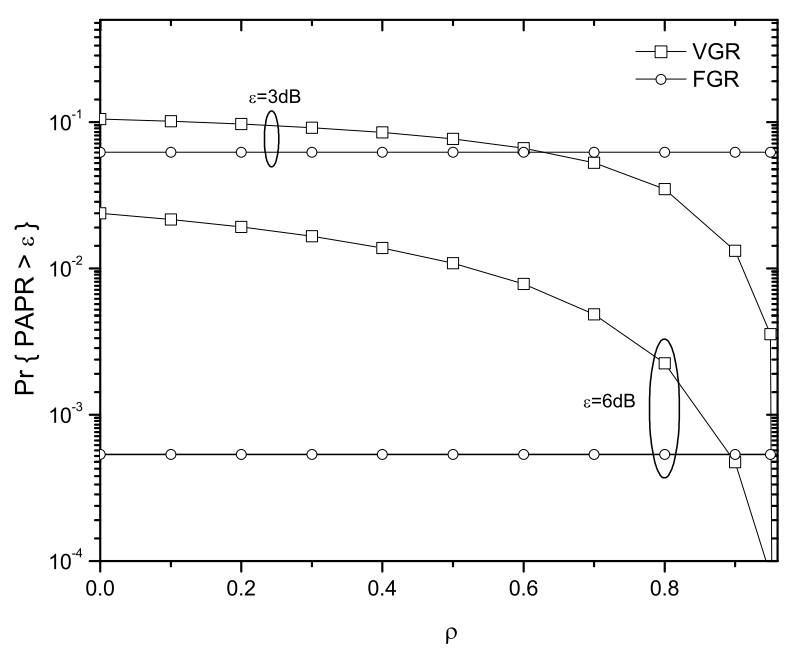

Fig. 9. CCDF of PAPR for VGR with outdated CSI and FGR vs. the correlation coefficient $\rho$, for $\varepsilon=3 \mathrm{~dB}$ and $\varepsilon=6 \mathrm{~dB}$, assuming $m_{X}=3$, $m_{Y}=1, \Omega_{X}=\Omega_{Y}=1$, and $\Gamma_{0}=25 \mathrm{~dB}$.

Fig. 8 illustrates the CCDF of the PAPR of VGR with outdated CSI, in comparison with that of FGR. The Nakagami$m$ parameter considered was $m_{X}=3$. Solid lines correspond to the upper bound of the CCDF of VGR, and were generated from (26). Dotted lines correspond to FGR, and were generated from (31). The simulated CCDF of the PAPR for VGR is also depicted, for transmit SNR $\Gamma_{0}=15 \mathrm{~dB}$ and $\Gamma_{0}=20 \mathrm{~dB}$, showing convergence to the upper bound for high SNRs. As expected, we notice a considerable increase in the probability of exceeding a certain value of PAPR for the VGR case, as $\rho$ decreases. For interpreting this result, let us consider the following example. Suppose that the linear region of the relay amplifier extends up to $\varepsilon=4 \mathrm{~dB}$ units higher than the ideal operation point. For perfect CSI $(\rho=1)$, the probability of exceeding the linear region tends to zero. However, for $\rho<1$ the probability of exceeding the linear region and thus distorting the output signal becomes non-zero, such that, for $\rho=0.8$ there exists $2 \%$ probability of exceeding the linear region, while for $\rho=0.2$ this probability is about $8 \%$. The latter fact implies that the reliability of the CSI at the relay is crucial for ensuring that the amplified signal is not distorted. From another viewpoint, if the CSI at the relay is not reliable enough one needs an amplifier with extended linear region, increasing thus its cost.

Finally, a comparison between VGR with outdated CSI and FGR, in terms of the CCDF of the PAPR, is shown in Fig. 9. Similarly to the comparison in terms of the ABEP, we notice that there exists a correlation coefficient threshold, $\rho_{0}$, such that for $\rho<\rho_{0}$ the CCDF of the PAPR is higher for VGR than for FGR. The higher the $\varepsilon$ value of interest (equivalently, the larger the linear region of the power amplifier at the relay), the higher $\rho_{0}$. Therefore, Fig. 9, in conjunction with Fig. 4, sheds some light on the required reliability of the CSI at the relay such that VGR is preferable over FGR.

\section{CONCLUSION}

When the gain used for channel compensation in $\mathrm{AF}$ variable gain relaying (a.k.a. CSI-assisted relaying) is based on imperfect CSI, the overall performance is considerably impaired. The performance degradation is the larger the lower the correlation between the actual and the estimated sourcerelay channel, yet such performance degradation does not affect the slope of the outage and error probability curves. This conclusion was drawn from a performance analysis for outdated CSI at the relay. Nevertheless, it was shown that the same analysis can also accommodate the case of Gaussian CSI errors at the relay. It was further observed that, depending on the maximum Doppler frequency, variable gain relaying requires frequent updates of the source-relay channel in order to outperform its less complex counterpart, that is, fixed gain relaying. Our investigation also included an analysis of the peak-to-average-power ratio for variable and fixed gain relaying, revealing that the probability of exceeding the amplifier's linear region (and thus causing signal distortion) can be higher for variable gain relaying, if the CSI at the relay is not reliable enough. An optimal power allocation analysis was also conducted and showed that the performance of $\mathrm{AF}$ relaying with outdated CSI at the relay can be substantially improved by appropriately dividing the power between the source and the relay terminal.

\section{REFERENCES}

[1] 3GPP TS36.912 V9.1.0: "Feasibility Study for Further Advancement for E-UTRA (LTE-Advanced)," 2010.

[2] J. N. Laneman, D. N. C. Tse, and G. W. Wornell, "Cooperative diversity in wireless networks: efficient protocols and outage behavior," IEEE Trans. Inf. Theory, vol. 50, pp. 3062-3080, Dec. 2004.

[3] G. Amarasuriya, C. Tellambura, and M. Ardakani, "Asymptoticallyexact performance bounds of AF multi-hop relaying over nakagami fading," IEEE Trans. Commun., vol. 59, pp. 962-967, Apr. 2011.

[4] H. A. Suraweera, P. J. Smith, A. Nallanathan, and J. S. Thompson, "Amplify-and-forward relaying with optimal and suboptimal transmit antenna selection,"' IEEE Trans. Wireless Commun., vol. 10, pp. 1874 1885, Jun. 2011.

[5] M. Gatzianas, L. Georgiadis, and G. K. Karagiannidis, "Gain adaptation policies for dual-hop nonregenerative relayed systems," IEEE Trans. Commun., vol. 55, pp. 1472-1477, Aug. 2007.

[6] P. Reynaert and M. Steyaert, RF Power Amplifiers for Mobile Communications. Springer, 2006.

[7] H. A. Suraweera, M. Soysa, C. Tellambura, and H. K. Garg, "Performance analysis of partial relay selection with feedback delay," IEEE Signal Process. Lett., vol. 17, pp. 531-534, Jun. 2010.

[8] G. Amarasuriya, C. Tellambura, and M. Ardakani, "Feedback delay effect on dual-hop MIMO AF relaying with antenna selection," Proc. 2010 IEEE Globecom.

[9] N. S. Ferdinand, N. Rajatheva, and M. Latva-aho, "Effects of feedback delay in partial relay selection over Nakagami-m fading chanels," IEEE Trans. Veh. Technol., vo. 61, pp. 1620-1634, May 2012.

[10] M. Torabi and D. Haccoun, "Capacity of amplify-and-forward selective relaying with adaptive transmission under outdated channel information," IEEE Trans. Veh. Technol., vol 60, pp. 2416-2422, Jun. 2011.

[11] J. L. Vicario, A. Bel, J. A. Lopez-Salcedo, and G. Seco, "Opportunistic relay selection with outdated CSI: outage probability and diversity analysis," IEEE Trans. Wireless Commun., vol. 8, pp. 2872-2876, Jun. 2009.

[12] M. Chen, T. C.-K. Liu, and X. Dong, "Opportunistic multiple relay selection with outdated channel state information," IEEE Trans. Veh. Technol., vol. 61, pp. 1333-1345, Mar. 2012.

[13] L. Fan, X. Lei, P. Fan, and R. Q. Hu, "Outage probability analysis and power allocation for two-way relay networks with user selection and outdated channel state information," IEEE Commun. Lett., vol. 16, pp. 638-640, May 2012.

[14] D. S. Michalopoulos, H. A. Suraweera, G. K. Karagiannidis, and R. Schober, "Amplify-and-forward relay selection with outdated channel estimates," IEEE Trans. Commun., vol. 60, pp. 1278-1289, May 2012.

[15] H. Phan, T. Q. Duong, M. Elkashlan, and H.-J. Zepernick, "Beamforming amplify-and-forward relay networks with feedback delay and interference," IEEE Signal Process. Lett., vol. 19, pp. 16-19, Jan. 2012. 
[16] K. Ishibashi and H. Ochiai, "Analysis of instantaneous power distributions for non-regenerative and regenerative relaying signals," IEEE Trans. Wireless Commun., vol. 11, pp. 258-265, Jan. 2012.

[17] B. Gedik and M. Uysal, "Impact of imperfect channel estimation on the performance of amplify-and-forward relaying," IEEE Trans. Wireless Commun., vol. 8, pp. 1468-1479, Mar. 2009.

[18] C. S. Patel and G. L. Stber, "Channel estimation for amplify and forward relay based cooperation diversity systems," IEEE Trans. Wireless Commun., vol. 6, pp. 2348-2356, June 2007.

[19] N. Yang, M. Elkashlan, J. Yuan, and T. Shen, "On the SER of fixed gain amplify-and-forward relaying with beamforming in Nakagami- $m$ fading," IEEE Commun. Lett., vol. 14, pp. 942-944, Oct. 2010.

[20] M. Nakagami, "The $m$-distribution-a general formula of intensity distribution of rapid fading," Statistical Methods Radio Wave Propag., vol. 40 , pp. $757-768$, Nov. 1962

[21] A. P. Prudnikov, Y. A. Brychkov, and O. I. Marichev, Integrals and Series, Vol. 4. Direct Laplace Transforms. Gordon and Breach Science Publishers, 1986.

[22] I. S. Gradshteyn and I. M. Ryzhik, Table of Integrals, Series and Products. Academic Press, 2000.

[23] A. Wojnar, "Unknown bounds on performance in Nakagami channels," IEEE Trans. Commun., vol. COM-34, no. 1, pp. 22-24, Jan. 1986.

[24] S. N. Datta and S. Chakrabarti, "Unified error analysis of dual-hop relay link in Nakagami- $m$ fading channels," IEEE Commun. Lett., vol. 14, no. 10, pp. 897-899, Oct. 2010.

[25] M. Abramowitz and I. A. Stegun, Handbook of Mathematical Functions with Formulas, Graphs, and Mathematical Tables, 9th edition. Dover, 1970.

[26] C. Jonietz, W. H. Gerstacker, and R. Schober, "Robust transmit processing for frequency-selective fading channels with imperfect channel feedback," IEEE Trans. Wireless Commun., vol. 7, pp. 5356-5368, Dec. 2008.

[27] G. K. Karagiannidis, N. C. Sagias, and T. A. Tsiftsis, "Exact closedform statistics for the sum of squared Nakagami-m variates and its applications," IEEE Trans. Commun., vol. 54, pp. 1353-1359, Aug. 2006.

[28] Y. Zhao, R. Adve, and T. J. Lim, "Symbol error rate of selection amplifyand- forward relay systems," IEEE Commun. Lett., vol. 10, pp. 757-759, Nov. 2006.

[29] http://functions.wolfram.com

[30] J. Zhang, T. Zhang, J. Huang, and R. Yuan, "ABEP of amplify-andforward cooperation in Nakagami- $m$ fading channels with arbitrary $m$," IEEE Trans. Commun., vol. 8, no. 9, pp. 4445-4449, Sep. 2009.

[31] W. C. Jakes, Microwave Mobile Communication. John Wiley \& Sons, 1974.

[32] Q. Liu, R. J. Baxley, X. Ma, and G. T. Zhou, "Error vector magnitude optimization for OFDM systems with a deterministic peak-to-average power ratio constraint," IEEE J. Sel. Top. Signal Process., vol. 3, no. 3, pp. 418-429, Jun. 2009.

[33] R. J. Baxley, B. Walkenhorst, and G. T. Zhou, "Signal classification using a peak-to-average power ratio statistic," 2009 IEEE International Conference on Communications.

[34] R. Zhao and L. Yang, "Performance analysis of fixed gain relaying systems in Nakagami-m fading channels." 2009 IEEE International Conference on Wireless Communications and Signal Processing.

[35] D. B. da Costa and M. B. Yacoub, "Dual-hop transmissions with semiblind relays over Nakagami- $m$ fading channels," Electron. Lett., vol.44, no. 3, pp. 214-216, Jan. 2008.

[36] H. Mheidat and M. Uysal, "Impact of receive diversity on the performance of amplify-and-forward relaying under APS and IPS power constraints," IEEE Commun. Lett., vol. 10, no. 6, pp. 468-470, Jun. 2006.

[37] H. A. Suraweera and G. K. Karagiannidis, "Closed-form error analysis of the non-identical Nakagami-m relay fading channel," IEEE Commun. Lett., vol. 12, no. 4, pp. 259-261, Apr. 2008.

[38] F. Xu, F. C. M. Lau, and D.-Wu Yue, "Diversity order for amplify-andforward dual-hop systems with fixed-gain relay under Nakagami fading channels," IEEE Trans. Wireless Commun., vol. 9, no. 1, pp. 92-98, Jan. 2010.

[39] M. Hasna and M.-S. Alouini, "Optimal power allocation for relayed transmissions over Rayleigh-Fading channels," IEEE Trans. Wireless Commun., vol. 3, no. 6, Nov. 2004.

[40] X. Deng and A. M. Haimovich, "Power allocation for cooperative relaying in wireless networks," IEEE Commun. Lett., vol. 9, no. 11, pp. 994-996, Nov. 2005.

[41] Y. Zhao, R. Adve, and T. J. Lim, "Improving amplify-and-forward relay networks: optimal power allocation versus selection," IEEE Trans. Wireless Commun., vol. 6, no. 8, pp. 3114-3123, Aug. 2007.
[42] S. Boyd and L. Vandenberghe, Convex Optimization. Cambridge University. Press, 2004

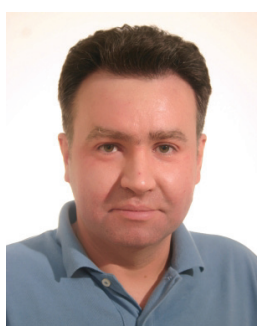

Zoran Hadzi-Velkov (M'97, SM'11) received the Dipl. Ing. (honors), M.Sc. (honors), and Ph.D. degrees in electrical engineering from the Ss. Cyril and Methodius University, Skopje, R. Macedonia, in 1996, 2000, and 2003, respectively. In 1996, he joined the Faculty of Electrical Engineering and Information Technologies, Ss. Cyril and Methodius University, where he is currently an Associate Professor. During 2001 and 2002, he was a visiting scholar at the International Business Machines (IBM) TJ Watson Research Center, Yorktown Heights, NY, USA. Since October 2012, Dr. Hadzi-Velkov is a visiting scientist at the Institute of Digital Communications (IDC), University of Erlangen-Nuremberg (FAU) in Germany. To realize this research stay, he was awarded the Alexander von Humboldt fellowship for experienced researchers. His current research interests are in the general area of digital communications over fading channels, with particular emphasis on cooperative communications, and energy-efficient communications.

Dr. Hadzi-Velkov currently serves as the Chair of the Republic of Macedonia Chapter of the IEEE Communications Society. He has served on the Technical Program Committees of several international conferences, including the IEEE ICC 2013. Since January 2012, Dr. Hadzi-Velkov is an Associate Editor for the IEEE COMMUNICATIONS LETTERS.

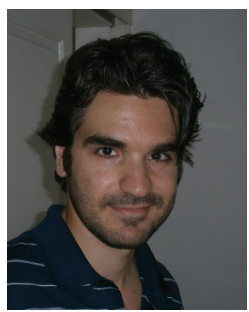

Diomidis S. Michalopoulos (S'05, M'10) was born in Thessaloniki, Greece, in 1983. He received the Diploma degree (5 years) and the $\mathrm{PhD}$ degree from the Electrical and Computer Engineering Department of the Aristotle University of Thessaloniki in 2005 and 2009, respectively. Since 2009, he is with the University of British Columbia, Canada, as a Killam postdoctoral fellow, and since 2011 , as a Banting postdoctoral fellow. His research interests span in the areas of wireless cooperative communications, communications theory, and free space

optical communications.

Dr. Michalopoulos received the Marconi Young Scholar award from the Marconi Society in 2010, the Killam postdoctoral fellow research prize for excellence in research in the University of British Columbia in 2011, and the Best Paper Award of the Wireless Communications Symposium (WCS) in the IEEE International Conference on Communications (ICC07). He has served as member of Technical Program Committees for IEEE conferences such as Globecom, WCNC, and VTC. He is also an Associate Editor of the IEEE COMMUNICATIONS LETTERS.

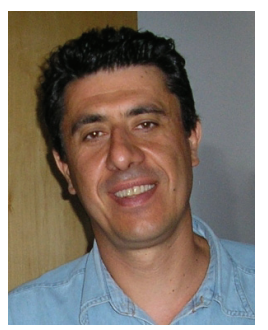

George K. Karagiannidis (SM'2003) was born in Pithagorion, Samos Island, Greece. He received the University Diploma (5 years) and Ph.D degree, both in electrical and computer engineering from the University of Patras, in 1987 and 1999, respectively. From 2000 to 2004, he was a Senior Researcher at the Institute for Space Applications and Remote Sensing, National Observatory of Athens, Greece. In June 2004, he joined the faculty of Aristotle University of Thessaloniki, Greece where he is currently Associate Professor (four-level academic rank system) of Digital Communications Systems at the Electrical and Computer Engineering Department and Head of the Telecommunications Systems and Networks Lab.

His research interests are in the broad area of digital communications systems with emphasis on communications theory, energy efficient MIMO and cooperative communications, cognitive radio and optical wireless communications.

$\mathrm{He}$ is the author or co-author of more than 180 technical papers published in scientific journals and presented at international conferences. He is also author of the Greek edition of a book on "Telecommunications Systems" and coauthor of the book Advanced Wireless Communications Systems (Cambridge Publications, 2012). He is co-recipient of the Best Paper Award of the Wireless Communications Symposium (WCS) in the IEEE International Conference on Communications (ICC'07), Glasgow, U.K., June 2007. 
Dr. Karagiannidis has been a member of Technical Program Committees for several IEEE conferences such as ICC, GLOBECOM, VTC, etc. In the past he was Editor for Fading Channels and Diversity of the IEEE TRANSACTIONS ON COMMUNICATIONS, Senior Editor of IEEE COMMUNICATIONS LETTERS and Editor of the EURASIP Journal of Wireless Communications \& Networks. He was Lead Guest Editor of the special issue on "Optical Wireless Communications" of the IEEE JOURNAL ON SELECTED AREAS IN COMmunications and Guest Editor of the special issue on "Large-scale multiple antenna wireless systems." Since January 2012, he is the Editor-in Chief of the IEEE COMMUNICATIONS LETTERS.

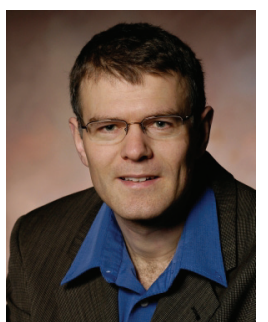

Robert Schober (S'98, M'01, SM'08, F'10) was born in Neuendettelsau, Germany, in 1971. He received the Diplom (Univ.) and the Ph.D. degrees in electrical engineering from the University of Erlangen-Nuermberg in 1997 and 2000, respectively. From May 2001 to April 2002 he was a Postdoctoral Fellow at the University of Toronto, Canada, sponsored by the German Academic Exchange Service (DAAD). Since May 2002 he has been with the University of British Columbia (UBC), Vancouver, Canada, where he is now a Full Professor. Since January 2012 he is an Alexander von Humboldt Professor and the Chair for Digital Communication at the Friedrich Alexander University (FAU), Erlangen, Germany. His research interests fall into the broad areas of Communication Theory, Wireless Communications, and Statistical Signal Processing.

Dr. Schober received several awards for his work including the 2002 Heinz MaierLeibnitz Award of the German Science Foundation (DFG), the 2004 Innovations Award of the Vodafone Foundation for Research in Mobile Communications, the 2006 UBC Killam Research Prize, the 2007 Wilhelm Friedrich Bessel Research Award of the Alexander von Humboldt Foundation, the 2008 Charles McDowell Award for Excellence in Research from UBC, a 2011 Alexander von Humboldt Professorship, and a 2012 NSERC E.W.R. Steacie Fellowship. In addition, he received best paper awards from the German Information Technology Society (ITG), the European Association for Signal, Speech and Image Processing (EURASIP), IEEE WCNC 2012, IEEE Globecom 2011, IEEE ICUWB 2006, the International Zurich Seminar on Broadband Communications, and European Wireless 2000. Dr. Schober is a Fellow of the Canadian Academy of Engineering and a Fellow of the Engineering Institute of Canada. He is currently the Editor-in-Chief of the IEEE TRANS ACTIONS ON COMMUNICATIONS. 